\title{
AN ORIFICE PLATE PULSE COLUMN FOR LIQUID-LIQUID EXTRACTION
}

\author{
By \\ David C. O'Brien \\ Submitted in Partial Fulfillment of the \\ Requirements for the Degree of Master \\ of Science from the Massachusetts \\ Institute of Technology \\ 1954
}

MASSACHUSETTS INSTITUTE OF TECHNOLOGY Enǵineerinǵ Practice School

\author{
Prepared From Work Performed Under Subcontract 70 \\ With The \\ CARBIDE AND CARBON CHEMICALS COMPANY \\ A Division of Union Carbide and Carbon Corporation \\ Oak Ridge, Tennessee
}

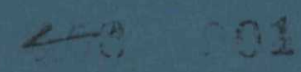




\section{DISCLAIMER}

Portions of this document may be illegible in electronic image products. Images are produced from the best available original document. 


\section{MASSACHUSETTS INSTITUTE OF TECHNOLOGY \\ ENGINEERING PRACTICE SCHOOL}

CARBIDE AND CARBON CHEMICALS COMPANY

A Division of Union Carbide and Carbon Corporotion

\section{MEMORANDUM}

EPS-K-209

$\mathrm{KT}-170$

August 23, 1954

TO:

J.C. Bresee

FROM:

D.C. O'Brien

SUBJECT: AN DRIFICE PLATE PULSE COLUMN FOR LIQUID-LIQUID EXTRACTION

DISTRIBUTION :

1. R.M. Batch

14. R.C. Olson

2. F.R. Bruce

15. J.H. Pashley

3. F.L. Culler

16. S.H. Smiley

4. W.K. Eister

17. G.A. Strasser

5. H.E. Goeller

18. B.H. Thompson

6. C.I. Gritzner

19. L.P. Twichell

7. J.M. Herndon

20. P.R. Vanstrum

8. H.K. Jackson

21. T.F. Wagner

9. A.C. Jealous

22. W.K. Whitson, Jr.

10. D.M. Lang

23. R.R. Lowery (K-25 RC)

11. E. Lieberman

24-26. R.R. Lowery (K-25 Plant Records)

12. J.A. Marshall

27-33. M.I.T. Practice School Files

13. G.W. Mitchel 
AN ORIFICE PLATE PULSE COLUMN FOR LIQUID-LIQUID EXTRACTION

by

David C. O'Brien

Submitted in Partial Fulfillment of the Requirements

for the Degree of

Master of Science

from the

Massachusetts Institute of Technology

1954

$\checkmark$

Professor in Charge of Research
Head of Department Author

Savid C. O'Brien 


\section{ACKNOWLEDGEMENTS}

The author is grateful to Carbide and Carbon Chemicals Company for furnishing the equipment, materials, laboratory, photographic and reproduction services which made this thesis possible. Appreciation is also due Professor James C. Bresee for the keen interest he has taken in this investigation. His constructive criticisms and encouragement always came at times when they were most needed.

The author also wishes to extend his thanks to Allan S. Hoffman for the time he spent proofreading and checking calculations. Alice Maxwell similarly deserves praise for her timely aid in the reproduction of this thesis. 
Submitted by David C. O'Brien on August 23, 1954, in partial fulfillment of the requirements for the degree of Master of science at the Massachusetts Institute of Technology.

A study was made of the performance of an orifice plate pulse column which is essentially a spray column containing internal constrictions in the form of orifices. The chemical system studied was acetic acid-waterhexone, and the variables considered were pulse frequency, throughput, phase continuity, and direction of solute transfer. For comparison purposes, several runs were made with a conventional spray column.

Curves are presented showing the effect of the column variables on the column efficiency (HTU). The trends observed are explained as being due principally to the competing effects of the area for mass transfer and back-mixing. Photographs of a typical column section show qualitatively the effect of the important column variables. 
I. SUMMARY . . . . . . . . . . . . . . . . . 7

II. INTRODUCTION ....................... 10

III. PROCEDURE . . . . . . . . . . . . . . . . 13

IV. RESULTS . . . . . . . . . . . . . . . . 19

V. DISCUSSION OF RESULTS

A. Precision and Accuracy of Results . . . . . . . . . 35

B. Factors Affecting HTU . . . . . . . . . . . . 37

C. Examination of HIU Results

1. Aqueous Phase Dispersed - Transfer into Organic Phase . . . 41

2. Organic Phase Dispersed - Transfer into Organic Phase . . 42

D. Flooding Range Curve ................ . . 43

E. Discussion of Overall Operation ................ 44

VI. CONCLUSIONS . . . . . . . . . . . . . . . 47

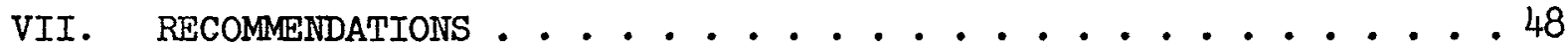
VIII. APPENDIX

A. Description of Apparatus ............... . 49

B. Chemical System and Analytical Methods . . . . . . . . 52

c. Details of Procedure . . . . . . . . . . . . 54

D. Sample Calculations . . . . . . . . . . 57

E. Sumary of Data and Calculated Values . . . . . . . 64

F. Table of Nomenclature . . . . . . . . . . . 67

G. Location of Original Data . . . . . . . . . 67

H. Literature Citations . . . . . . . . . . 68 
LIST OF FIGURES AND TABLES

Table Number

$\begin{array}{rlr}\text { I } & \text { Preliminary Extraction Runs (Aqueous Discontinuous) } & 33 \\ \text { II } & \text { Stripping Runs (Organic Discontinuous) } & 34 \\ \text { III } & \text { Equilibrium Data (Acetic Acid, Water, Hexone System) } & 53 \\ \text { IV } & \text { Summary of Data and Calculated Values }\end{array}$

Figure Number

$1 \quad$ Schematic Flowsheet - Orifice Plate Pulse Column

$2 \quad$ Orifice Plate Pulse Column and Auxiliaries 15

$3 \quad$ Closeup of Pulsing Apparatus 16

$\begin{array}{lll}4 & \text { Spray Tower } & 17\end{array}$

5 Effect of Pulse on HTU and Holdup 20

6 Effect of Pulse on HTU and Holdup 21

$7 \quad$ Effect of Pulse on HIU and Holdup 22

8 Effect of Pulse on Orifice Plate Pulse Column Operation aqueous discontimuous , 23

9 Effect of Pulse on Orifice Plate Pulse Column Operation organic discontinuous

10 Effect of Pulse on HIU and Holdup

$320 \mathrm{gm} . / \mathrm{min}$. solute-free aqueous flow 25

11 Effect of Pulse on HIU and Holdup
$400 \mathrm{gm} . / \mathrm{min}$. solute-free aqueous flow

12 Effect of Phase Continuity on Orifice Plate Pulse $\begin{array}{ll}\text { Column Operation } & 27\end{array}$

13 Effect of Throughput on Orifice Plate Pulse Column Operation - aqueous discontinuous 28

14 Effect of Throughput on Orifice Plate Pulse Column Operation - organic discontinuous

$$
\therefore \quad .06
$$


Figure Number (Cont'd)

15 Flooding hange for Orifice Plate Pulse Colurn

16 Flooding in an Orifice Plate Pulse Column organic discontinuous

31

17 Effect of Throughput and Pulse on Spray Tower Operation - organic discontinuous 


\section{SUMMARY}

During the past ten years liquid-liquid extraction has become one of the most important unit operations in the field of chemical engineering. Along with the development of new chemical systems, extraction devices also have been improved. The pulse column is an outstanding example of an improvement of vertical extraction columns. Investigations have shown that the addition of a low frequency pulse to a sieve plate column will increase the extraction efficiency while maintaining a high column throughput.

Previous investigators, interested in utilizing the advantages of the high throughputs afforded by spray towers, studied the effect of pulsing on spray tower operation. These investigators concluded that the effect of pulsing a spray tower was slight and that improvement could be obtained only by incorporating internal constrictions into the spray tower design. It was suggested that regularly spaced orifices would provide the necessary constrictions and yet would allow throughputs higher than those attainable in sieve plate pulse columns. Also, there might be cases where an orifice plate pulse column would have decided advantages over a sieve plate pulse column, such as for extraction from slurries.

The orifice plate pulse column used in this investigation consisted of individual sections of Pyrex pipe, 4 inches long with an internal diameter of 1-1/2 inches, fitted together with flanges and gaskets. The gaskets served as orifices and constricted the area for flow to 25 per cent of the column cross-sectional area. The purpose of this investigation was twofold:

1) To determine the effect of pulse frequency on extraction efficiency and discontinuous phase holdup over a range of throughputs, and

2) To determine the effect of pulse frequency on the maximum throughput attainable in an orifice plate pulse column.

The chemical system employed for this investigation was acetic acid, water and methyl-isobutyl ketone. Most of the data were taken with transfer of acetic acid into the organic phase (termed extraction) with the aqueous 
phase dispersed. Aqueous feed concentration for the extraction runs was maintained approximately constant and equal to $14 \mathrm{wt}$. per cent acetic acid. Some data were also obtained with the organic phase discontinuous, again for extraction runs. Other runs were made on the regeneration of organic solvent (termed stripping) with the aqueous phase dispersed. Five extraction runs were made with a spray tower (a single straight section substituted for the orifice sections) with the ketone as the dispersed phase.

For transfer of acetic acid into the organic phase, solute-free aqueous flow rates ranged from 75 to 400 grams per minute. The ratio of organic flow to aqueous flow was held approximately constant at 1.3 gms. organic/gm. aqueous (on a solute-free basis). Pulse height was also held constant throughout the investigation at a value of $1 / 2$ inch. Frequencies were varied from 0 to 200 cycles per minute, with the exception of one spray tower run which was pulsed at 300 cycles per minute. Photographs were taken of orifice plate pulse column operation to show changes in drop size, holdup and dispersion.

Extraction efficiencies were expressed in terms of overall HIU's, calculated from log mean driving forces. HIU values for extraction runs varied from 35 to 20 inches at low throughputs and from 30 to 13 inches at high throughputs. The lowest HTU values were obtained at high frequencies. One spray tower run at intermediate flow rates and 300 cycles per minute resulted in an HTU of 11 inches. The flooding range curve exhibited a maximum at 100 cycles per minute. Combined flow rates at flooding varied from $550 \mathrm{gm} . / \mathrm{min}$. (solute-free basis) at 0 and 180 cycles per minute to $1200 \mathrm{gm} . / \mathrm{min}$. at the maximum.

On the basis of the results of this investigation it was concluded that: (1) pulsing tended to increase holdup and improve extraction efficiency, particularly at high frequencies and at high throughputs, (2) pulsing tended to create smaller drops and increase back-mixing, particularly at low throughputs, (3) an increase in throughput tended to increase holdup and improve extraction efficiency, particularly at 'high frequencies, and, (4) an increase in throughput tended to increase drop size and decrease back-mixing.

$$
\therefore 99
$$


It was further concluded that (5) dispersion of the organic phase in preference to the aqueous phase, when solute transfer is into the organic phase, resulted in more holdup and increased transfer area. These factors increased the extraction efficiency at high flow rates and low frequencies. In addition, (6) pulsing in an orifice plate pulse column tended to increase maximum throughput by pumping action and to decrease maximum throughput by increasing holdup.

In view of the effects of pulsing in an orifice plate pulse column and the possibility that the potential of the spray tower as an extraction device may be increased by pulsing, it is recommended that: (1) a more detailed study be made on an orifice plate pulse column to determine the effect of frequency on average drop size, back-mixing and coalescence, perhaps with the aid of motion pictures and dyes, (2) studies be made to determine the effect of pulse height, cam shape and orifice size, shape and spacing on HIU, the factors influencing HIU, and flooding, and (3) studies also be made to determine the effect of pulsing on spray tower operation and extraction efficiency, with particular emphasis on the mechanism of dispersion and the effect of varied inlet design. 


\section{INTRODUCTION}

Continuous countercurrent liquid-liquid extraction is one of the most fertile areas for chemical engineering research today. Although horizontal contactors and mixer-settler techniques are being developed, a major portion of solvent extraction is done in vertical columns (4). The latter's importance is evidenced by increased usage as well as the growing efforts of investigators to improve spray and packed tower operation.

Improvements may be classified in three categories: greater extraction efficiency (as measured by HIU, HETS, or per cent recovery), higher throughput and increased versatility. Considerable attention has been devoted to the development of better packing and the addition of mechanical agitators to columns in order to increase the available area for mass transfer. Although packing and other internal additions improve the extraction efficiency, they frequently have detrimental effects on throughput and versatility. Recent developments, however, have shown that applying a pulse to a packed column, or a column fitted with sieve plates, increases the extraction efficiency while maintaining a high column throughput (2, 4 , 11).

Some of the preliminary sieve plate pulse column work was performed at the M.I.T. Engineering Practice School at Oak Ridge (1, $\underline{2}, \underline{6}, \underline{8}, \underline{10})$. In conjunction with this work it was suggested that high frequency pulsing of a spray column might favorably affect its operation as a contacting device. In November, 1952, two M.I.T. students, R.M. Lurie and R.G. Shaver, attempted to determine the effect of high frequency pulsing (7-12 cycles per second) on a spray column (7). Most of their work was done on a spray column constructed from glass sections which were joined at three points with flanges and gaskets. Through an oversight, the gaskets at these points constricted the cross-sectional area for flow to $63 \%$ of the column cross-section. It was found that pulsing this column improved extraction efficiency, lowering HIJ by as much as a factor of three. Later, upon discovery of the constrictions it was realized that the results had been biased. The constricting gaskets were removed and replaced with gaskets of the proper diameter. The investigators observed 
little improvement in dispersion and extraction efficiency relative to spray tower operation when this second column was pulsed. It therefore was concluded that pulsing would not improve spray column operation unless some constriction of flow was present.

The first column which Lurie and Shaver had constructed was basically a crude orifice plate pulse column. The orifice plate pulse column differs only slightly from the sieve plate pulse column in that the many small holes of the sieve plate are replaced by one large orifice. At the outset, such a column would appear to have several inherent advantages. Extraction efficiency would be improved in comparison to the spray tower, as observed by Lurie and Shaver (1). It also seems that the substitution of an orifice would increase the maximum throughput over that of a sieve plate pulse column under similar operating conditions. Furthermore, a single large hole would probably allow extraction from slurries, an operation in which plugging would pose a serious problem, especially in packed or sieve plate pulse columns. Construction and maintenance costs would probably be less than for a sieve plate pulse column.

In September, 1953, Myers, et. al. (9) constructed an orifice plate pulse column which successfully extracted material from a slurry. The report stated that a similar attempt by other investigators using mixersettler equipment resulted in failure. In May, 1954, Buchanan, et. al. (3) costructed a similar column which achieved extraction efficiencies comparable to those of a sieve plate pulse column operating with the same system under the same flow conditions. The extraction efficiencies of the orifice plate pulse column were obtained at higher frequencies, but the maximum throughput was observed to be greater than for a sieve plate pulse column operating with the same pulse.

Important operating variables of an orifice plate pulse column are pulse height, pulse frequency, wave shape, and flow rates. Design variables include orifice size, column diameter, plate spacing and the nature of the chemical system. Data from Myers, et. al. (9) were on a complex chemical system and offered little aid in determining the effects of the above variables on extraction efficiency and maximum throughput. 
Data from the latter project were not available at the start of this investigation.

Acetic acid, water, and methyl-isobutyl ketone (hexone), a chemical system used by many previous researchers, was employed in this investigation ( $\underline{1}, \underline{2}, \underline{5}, \underline{6}, \underline{7}, \underline{8}, \underline{11})$. This work constitutes a preliminary examination of pulse column variables. Its primary purpose was two-fold:

1) To study the effect of pulse on extraction efficiencies over a range of column throughputs.

2) To study the effect of pulse on the maximum throughput and holdup of the discontinuous phase.

Experimental work was conducted from March through July, 1954, at the Massachusetts Institute of Technology Engineering Practice School at Oak Ridge, Tennessee. 


\section{PROCEDURE}

The orifice plate pulse column used in this investigation was $1-1 / 2$ inches in diameter and contained 19 orifices spaced 4 inches apart. The orifice diameter was $3 / 4$ inches, thus reducing the area for flow to 25 per cent of its normal value. The important auxiliary equipment consisted of a pulsing unit, Saran tubing, valves and several storage tanks. Three of these tanks were used as feed pumps by utilizing air as a displacement medium (See Appendix, A. Details of Apparatus). Figure $I$ is a schematic flow diagram of the system and Figure 2 is a photograph of the equipment. Figure 3 is a closeup of the pulsing apparatus. All runs except five were made with the orifice plate pulse column. These five runs were made with a spray tower of the same dimensions (See Figure 4 ).

The major portion of the data were obtained for the transfer of acetic acid from water to hexone (methyl-isobutyl ketone). These runs were called extractions muns and the runs with reversed transfer were called stripping runs. For extraction runs, the aqueous feed concentration was held roughly constant at 14 per cent acetic acid by weight; the organic feed contained relatively little acid, usually 0.3 per cent or less.

At the start of a run, the pulsing unit was turned on and was set at the desired frequency. Frequencies ranged from 0 to 200 cycles per minute. Pulse amplitude in the column was $1 / 2$ inches and was the same throughout the investigation. The pulse pattern was essentially a sine wave.

After adjusting the air pressure on the displacement pumps, flow of the continuous phase (usually organic) was then fixed at the operating level, and the interface was placed midway between the continuous phase inlet and the discontinuous phase outlet. The interface was controlled by varying the aqueous phase outflow with an overflow gravity leg. Flow of the discontinuous phase was then started and the interface was readjusted until the operation was stable.

Flows were controlled with needle valves and were set with the aid of rotameters. The majority of flow measurements were made on the outlet 


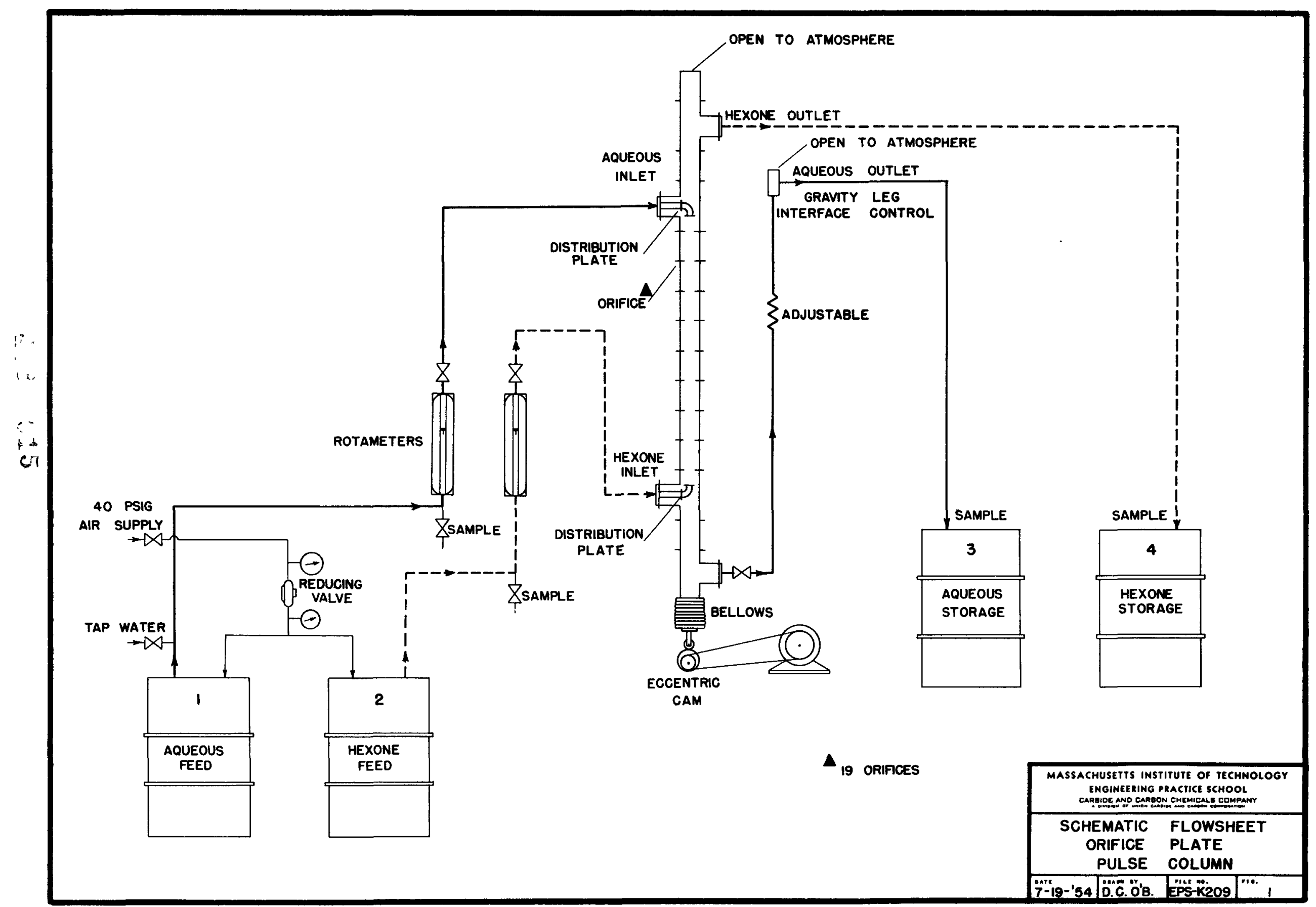




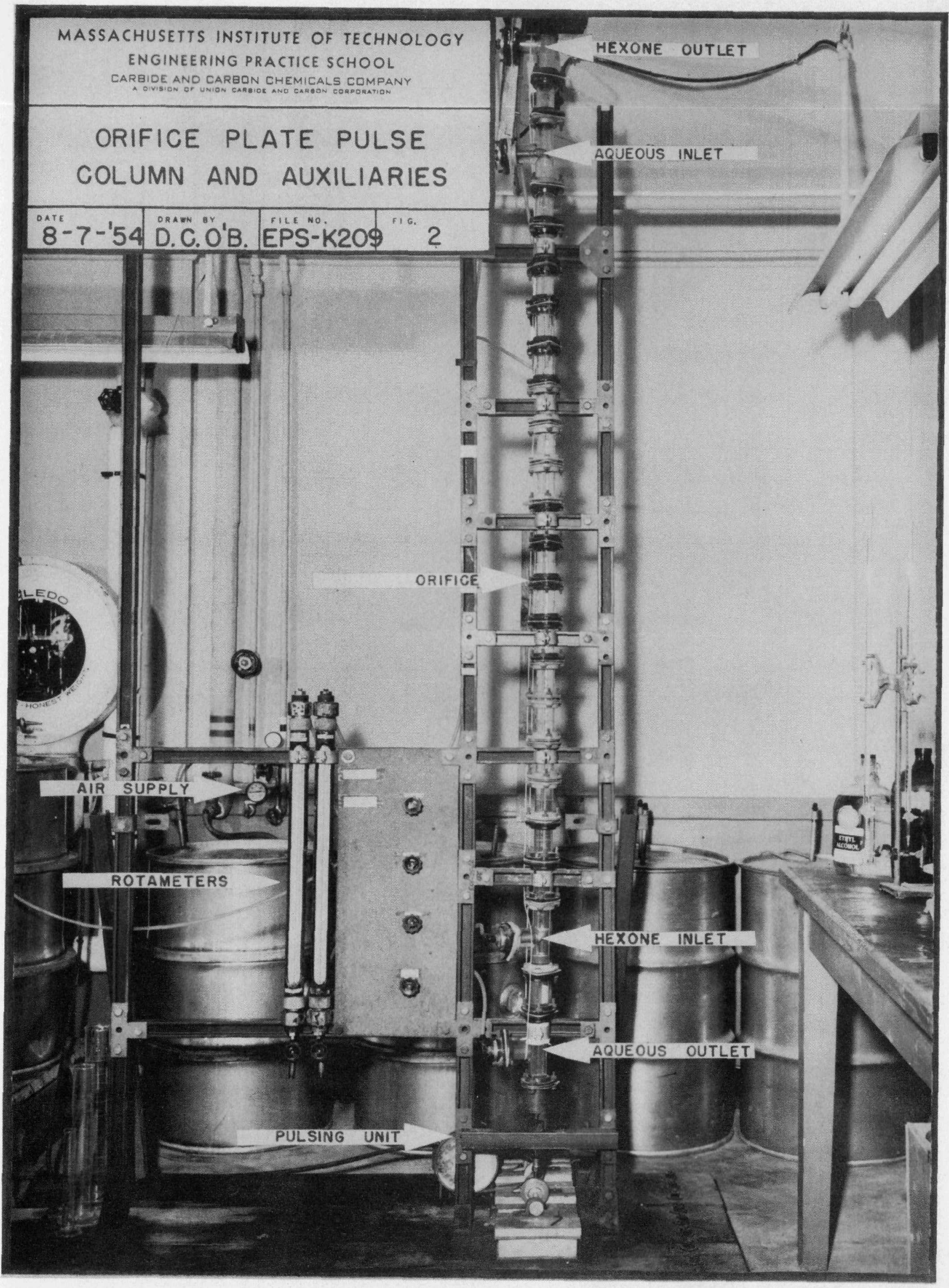




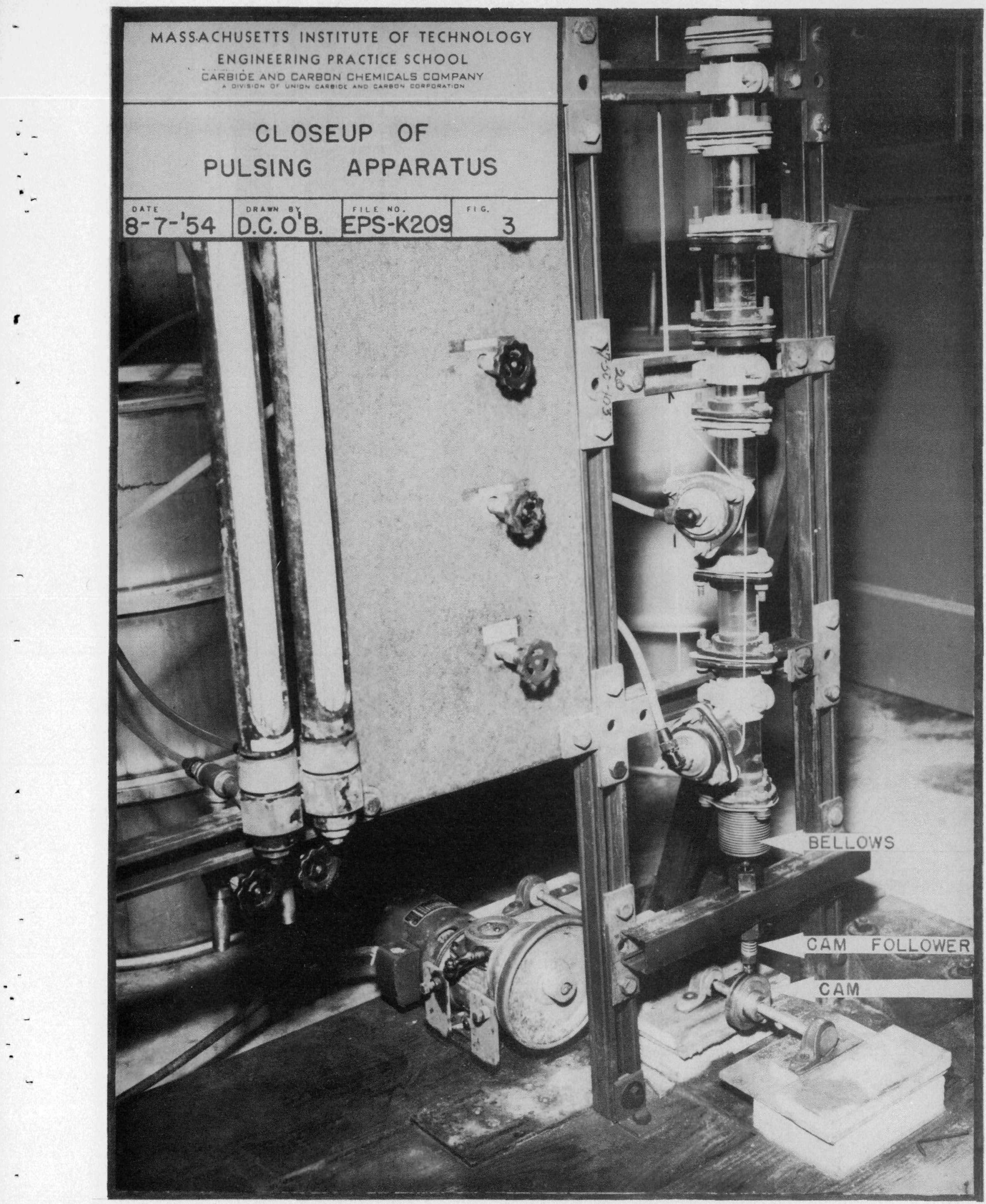




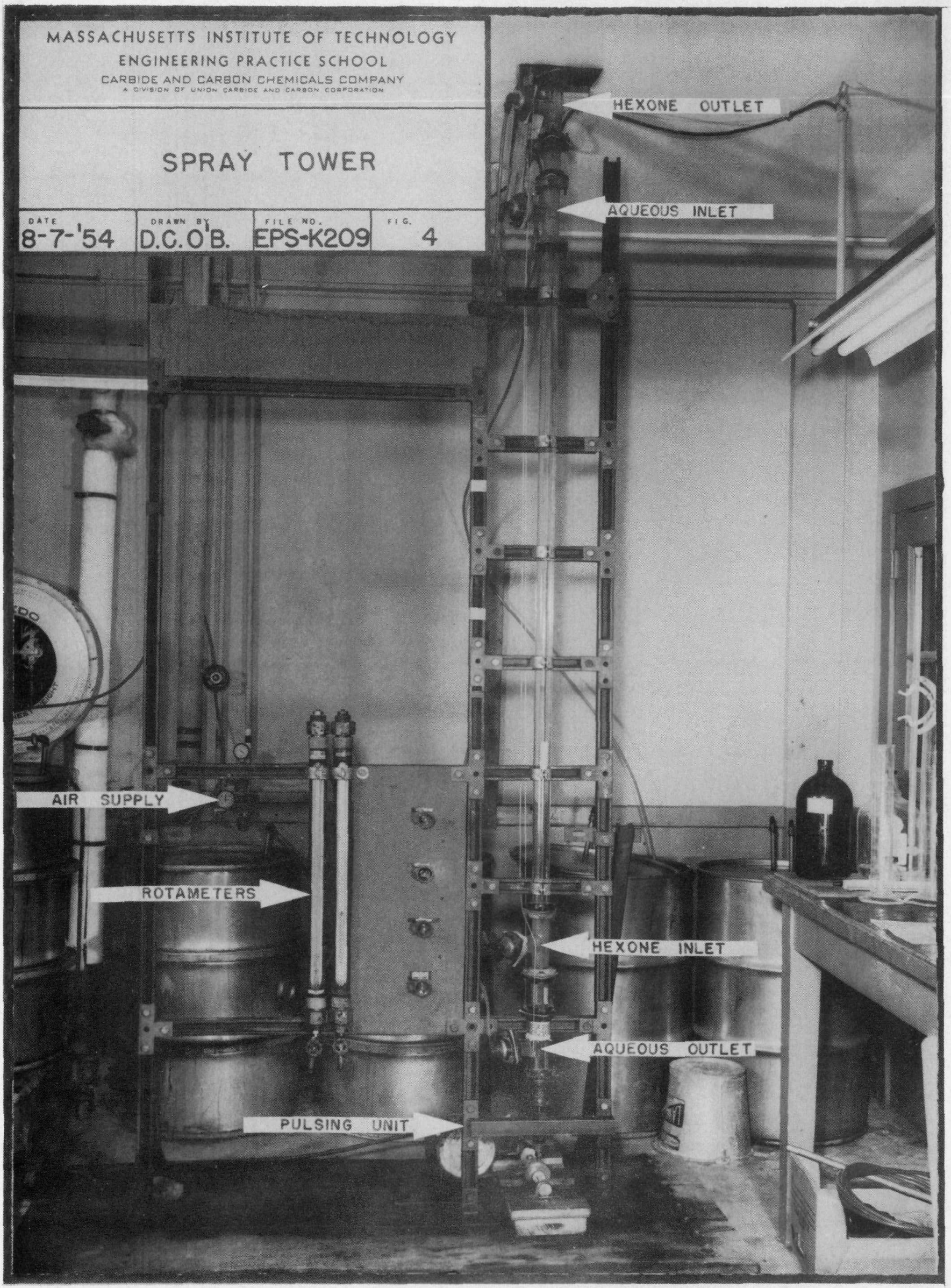


streams with a graduated cylinder and a stopwatch. Aqueous flows ranged from 75 - $400 \mathrm{gm}$. solute free solvent/min. The phase flow ratio was kept relatively constant at $1.3 \mathrm{gm}$. of solute free organic per gm. solute free aqueous.

The length of a run varied with the throughput and was roughly equal to the time required for six or seven changes of the continuous phase. Two samples, spaced thirty minutes apart, of both outlet streams were taken after the column had reached steady state. After the last samples were taken, flows were stopped and the two phases were allowed to separate. The interface was then returned to the operating position, and the overflow was collected in a cylinder. The overflow was equal to the discontinuous phase holdup.

The acid-rich hexone from extraction muns was stored and used as feed for stripping runs. Acid-depleted raffinate from extraction runs was discarded. The aqueous feed for stripping runs was tap water. Stripped hexone and the acid-rich aqueous product were reused as makeup for extraction run feeds.

Samples from all streams were analyzed for acid content by titration with sodium hydroxide to a phenolphthalein end point. The specific gravities of the samples were determined with hydrometers (See Appendix, B. Chemical System and Analytical Methods). Efficiencies were calculated from the concentrations in the form of HIU oa (Height of a Transfer Unit, Overall, based on the aqueous phase). The effective height for extraction was assumed to be 85 inches.

Flooding was investigated at several frequencies for both aqueous and organic phases discontinuous. Approximately 15 minutes was allowed to determine if the column was flooded at any particular throughput. Throughputs were varied from low to high at discrete intervals. Aqueous discontinuous flooding occurred when the aqueous phase could not pass entirely through the first orifice and thus was partially pushed out the top of the column. Organic discontinuous flooding was defined as the throughput at which coalescence of the drops occurred tending to make the discontinuous phase continuous. 
IV. RESULTS

The results of this investigation are presented in Figures $5-17$ and Tables I and II. All results presented in the figures are for extraction runs. Figures 5, 6, 7, 10, and 11 respectively correspond to solute free aqueous flows of $75,160,240,320$, and $400 \mathrm{gm} . / \mathrm{min}$. and are plots showing HTU ${ }_{0 O}$, HTU ${ }_{O a}$ and per cent discontinuous phase holdup over a frequency range of $0-180$ cycles/min. These plots also show individual spray tower runs as well as runs which compare aqueous discontinuous runs with organic discontinuous runs.

Figures 8 and 9 are photographic studies of runs presented in Figure 7. Figures 12, 13, and 14 are photographs illustrating the effect on HTU of throughput and the reversal of phase continuity. Figure 15 is a plot of the flooding range of the orifice plate pulse column as a function of frequency, and Figure 16 is a photograph of organic discontinuous phase flooding. Figure 17 pictorially illustrates the five spray tower runs.

Table I lists the important values for the preliminary extraction runs at 30 cycles/min. A similar listing for the stripping runs is given in Table II. 


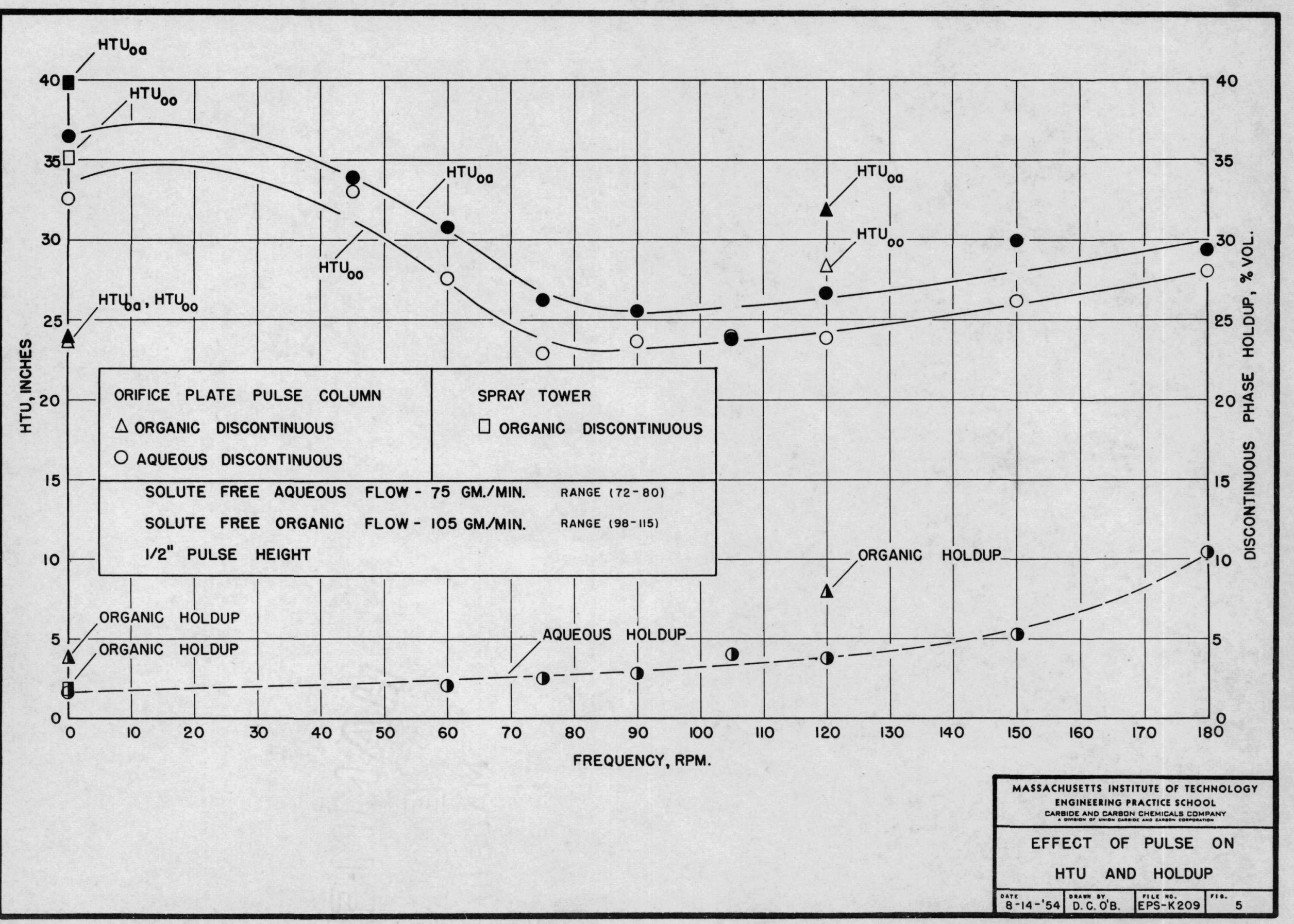




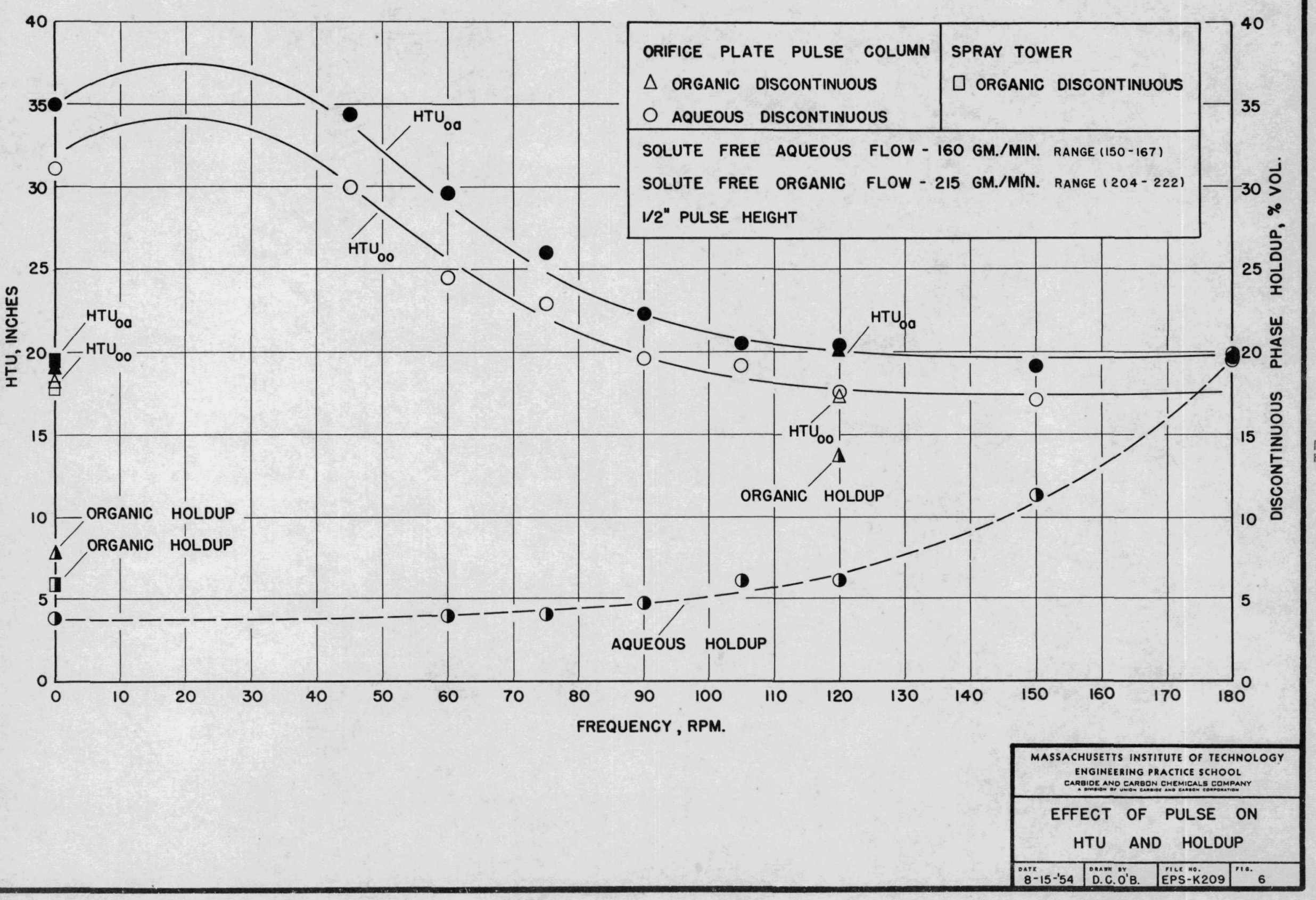




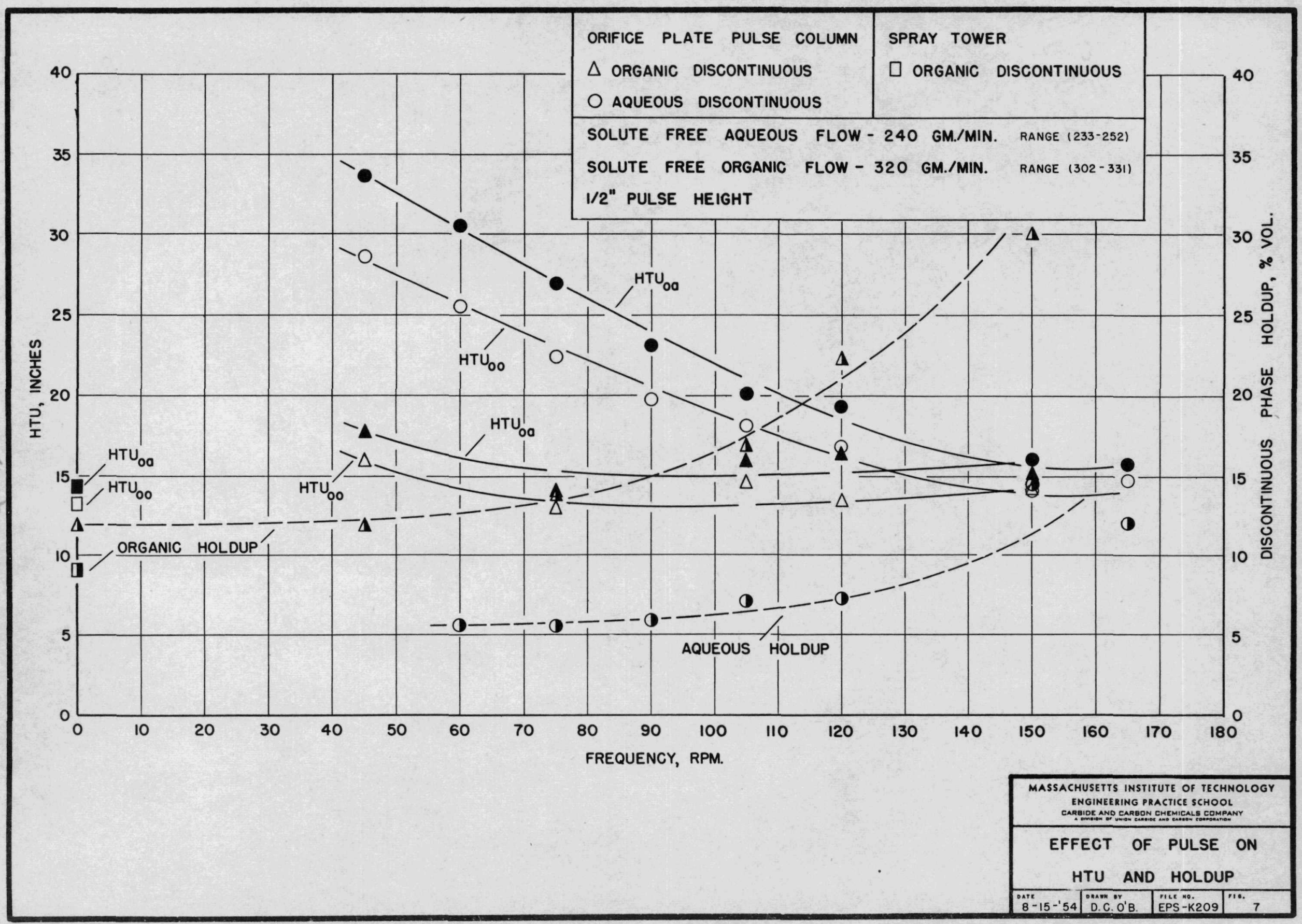




\section{OF PULSE ON ORIFICE PLATE PULSE COLUMN OPERATION}

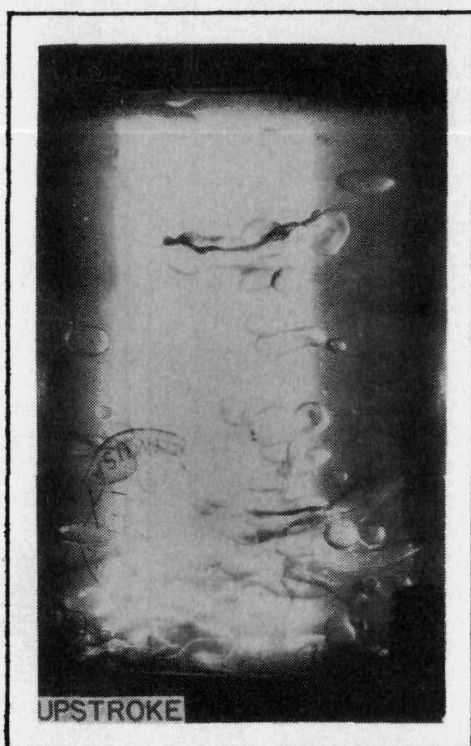

AQUEOUS FLOW - 240 GM./MIN. TOTAL FLOW - 560 GM./MIN. FREQ.- 45 RPM., RUN 13 VOLUME HOLDUP-(N.D.) HTU ${ }_{O O}-28.7^{\prime \prime} \quad H T U_{O a}-33.7^{\prime \prime}$

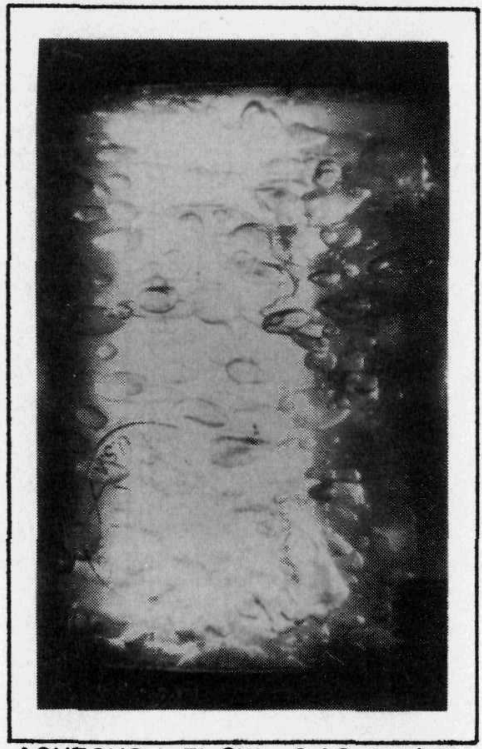

AQUEOUS FLOW- $240 \mathrm{GM} . / \mathrm{MIN}$. TOTAL FLOW-560 GM./MIN. FREQ.- 105 RPM., RUN 37 VOLUME HOLDUP-7.2\%

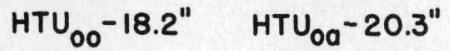

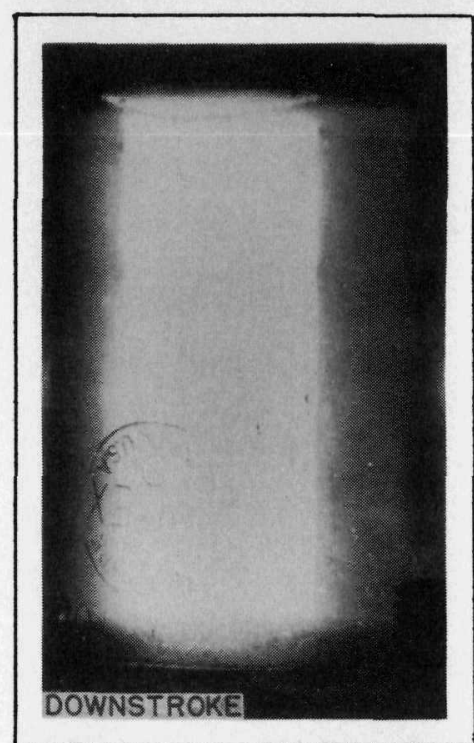

AQUEOUS FLOW- 240 GM./MIN. TOTAL FLOW-560 GM./MIN. FREQ.- 45 RPM., RUN 13

VOLUME HOLDUP-(N.D.)

HTU $\mathrm{OO}^{-28.7^{\prime \prime}} \quad \mathrm{HTU}_{\mathrm{OO}}{ }^{-33.7^{\prime \prime}}$

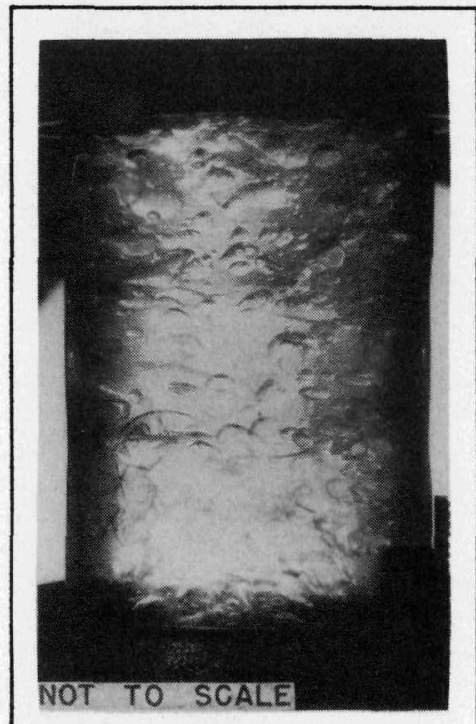

AQUEOUS FLOW- 240 GM./MIN. TOTAL FLOW- 560 GM./MIN. FREQ.- 120 RPM., RUN 43 VOLUME HOLDUP- $7.4 \%$ HTU $00^{-16.9^{\prime \prime}}$ HTU $_{0 a^{-19.4 "}}$

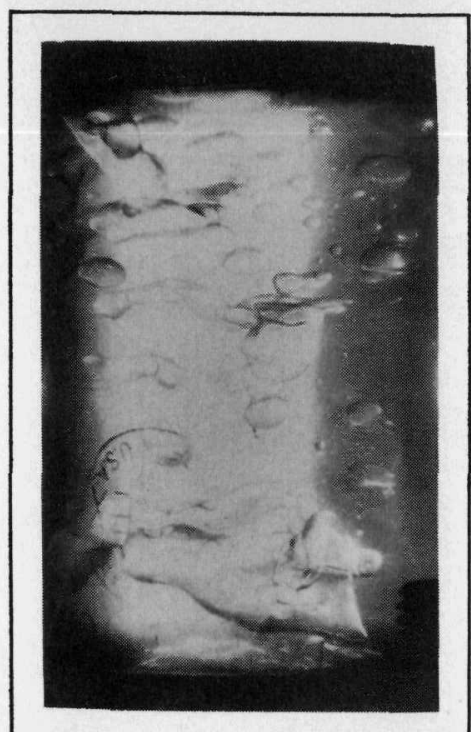

AQUEOUS FLOW-240 GM./MIN. TOTAL FLOW - 560 GM./MIN. FREQ.- 75 RPM., RUN 25 VOLUME HOLDUP- $5.6 \%$ $\mathrm{HTU}_{00}-22.5^{\prime \prime} \quad \mathrm{HTU}_{\mathrm{Oa}}-27$.

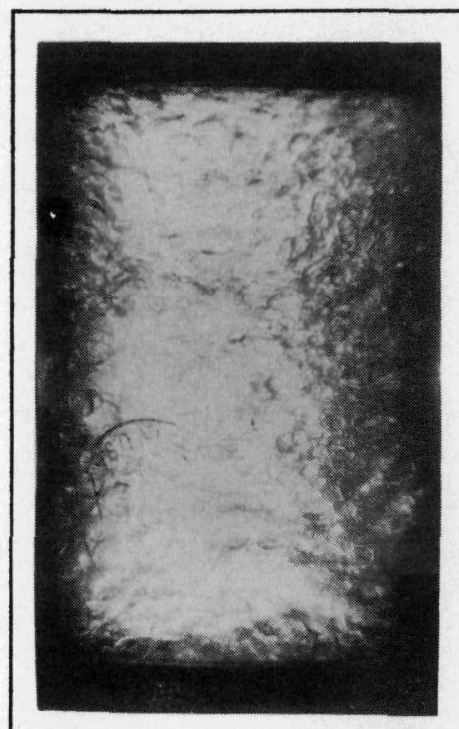

AQUEOUS FLOW- 240 GM./MIN. TOTAL FLOW- 560 GM./MIN.

FREQ. - 150 RPM., RUN 49

VOLUME HOLDUP-14.5\% HTU OO $^{-14.2^{11}}$ HTU OQ $^{-16.1^{\prime \prime}}$

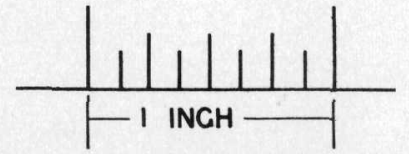

AQUEOUS DISCONTINUOUS

1/2" PULSE HEIGHT

FLOWS ON SOLUTE FREE BASIS
MASSACHUSETTS INSTITUTE OF TECHNOLOGY ENGINEERING PRACTICE SCHOOL CAREIDE AND EAREON CHEMICALS CDMPAN

ORIFICE PLATE PULSE COLUMN STUDIES

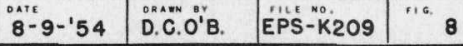


EFFECT OF PULSE ON ORIFICE PLATE PULSE COLUMN OPERATION

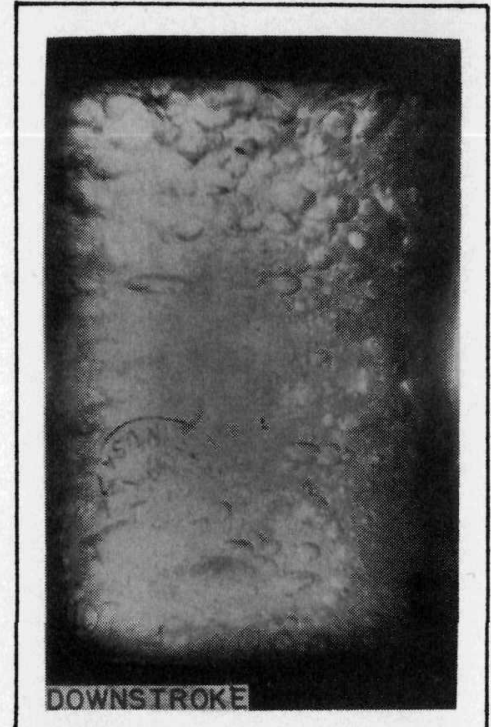

AQUEOUS FLOW- 240 GM./MIN. TOTAL FLOW - 560 GM./MIN. FREQ. - 45 RPM. , RUN 67 VOLUME HOLDUP- $12.0 \%$ HTU ${ }^{-16.0^{\prime \prime}}$ HTU $\mathrm{OO}^{-17.8^{\prime \prime}}$

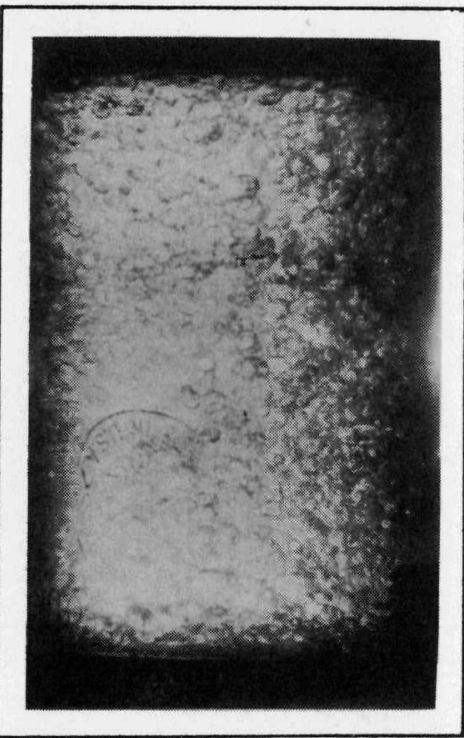

AQUEOUS FLOW - 240 GM./MIN. TOTAL FLOW- 560 GM./MIN. FREQ.- 105 RPM., RUN 69 VOLUME HOLDUP - $17.0 \%$ HTU $00^{-14.6 "}$ HTU $00^{-15.8 "}$

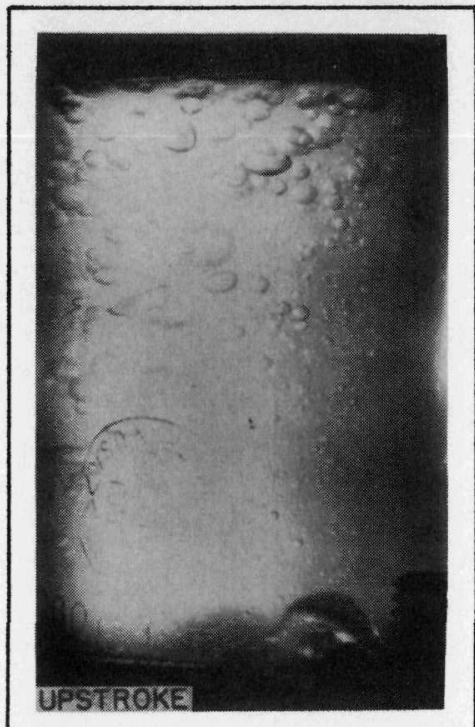

AQUEOUS FLOW- 240 GM./MIN. TOTAL FLOW- 560 GM./MIN. FREQ.- 45 RPM., RUN 67 VOLUME HOLDUP- $12.0 \%$ $\mathrm{HTU}_{00}{ }^{-16.0^{\prime \prime}} \mathrm{HTU}_{\mathrm{Oa}}{ }^{-17.8^{\prime \prime}}$

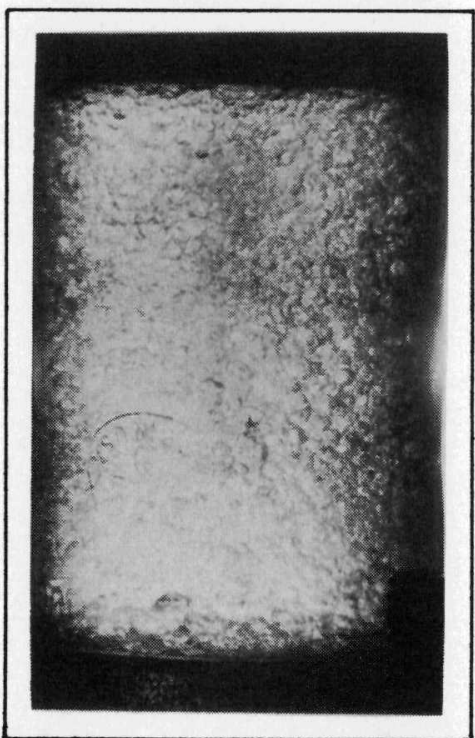

AQUEOUS FLOW - 240 GM./MIN TOTAL FLOW - 560 GM./MIN. FREQ.-120 RPM., RUN 64 VOLUME HOLDUP - $22.3 \%$

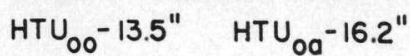

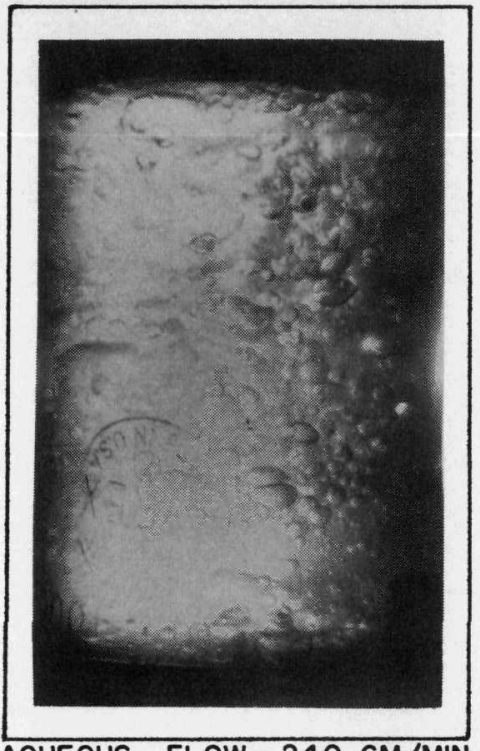

AQUEOUS FLOW-240 GM./MIN TOTAL FLOW - 560 GM./MIN. FREQ. -75 RPM., RUN 68 VOLUME HOLDUP $-13.9 \%$ HTU OO $^{-13.0^{\prime \prime}}$ HTU 0 Ō 14.2"

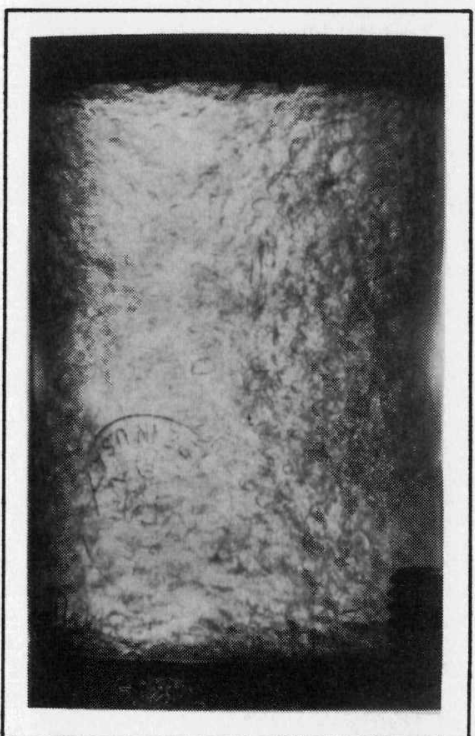

AQUEOUS FLOW - 240 GM./MIN. TOTAL FLOW- 560 GM./MIN. FREQ.- 150 RPM., RUN 70 VOLUME HOLDUP-30.1\% HTU OO- 14.1" HTU OO-15.3"

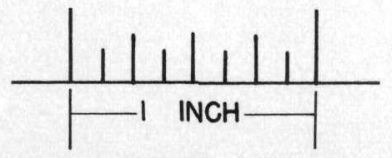

HEXONE DISCONTINUOUS

$1 / 2$ " PULSE HEIGHT

FLOWS ON SOLUTE FREE BASIS

MASSACHUSETTS INSTITUTE OF TECHNOLOGY ENGINEERING PRACTICE SCHOOL CARBIDE AND CAPBON GHEMESCHOMPAN

ORIFICE PLATE PULSE COLUMN STUDIES

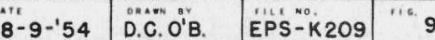




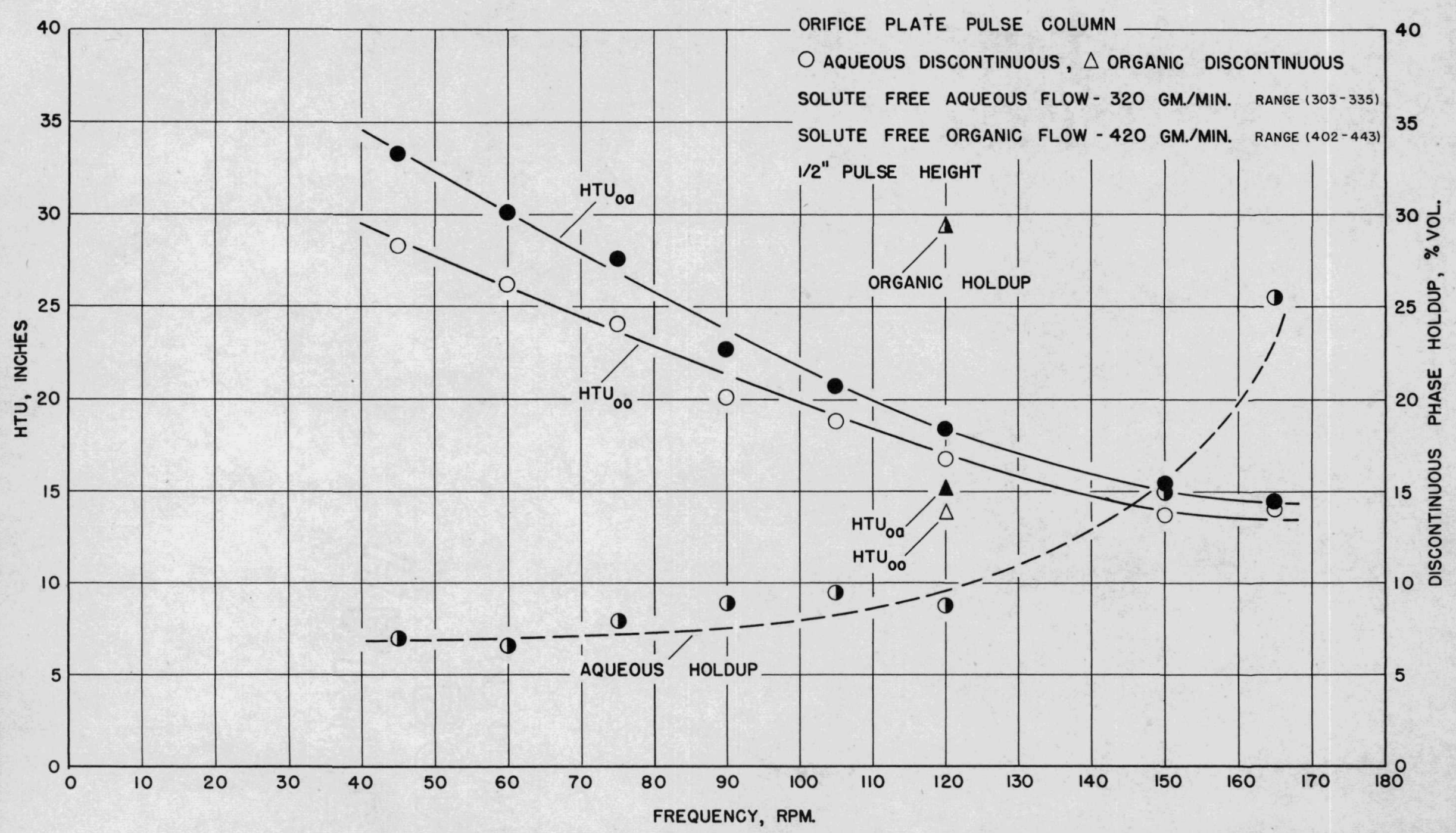

MASSACHUSETTS INSTITUTE OF TECHNOLOGY ENGINEERING PRACTICE SCHOOL
CARGIOE ANO GARBON CHEMICALS COMPANY

EFFECT OF PULSE ON

HTU AND HOLDUP

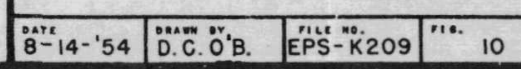




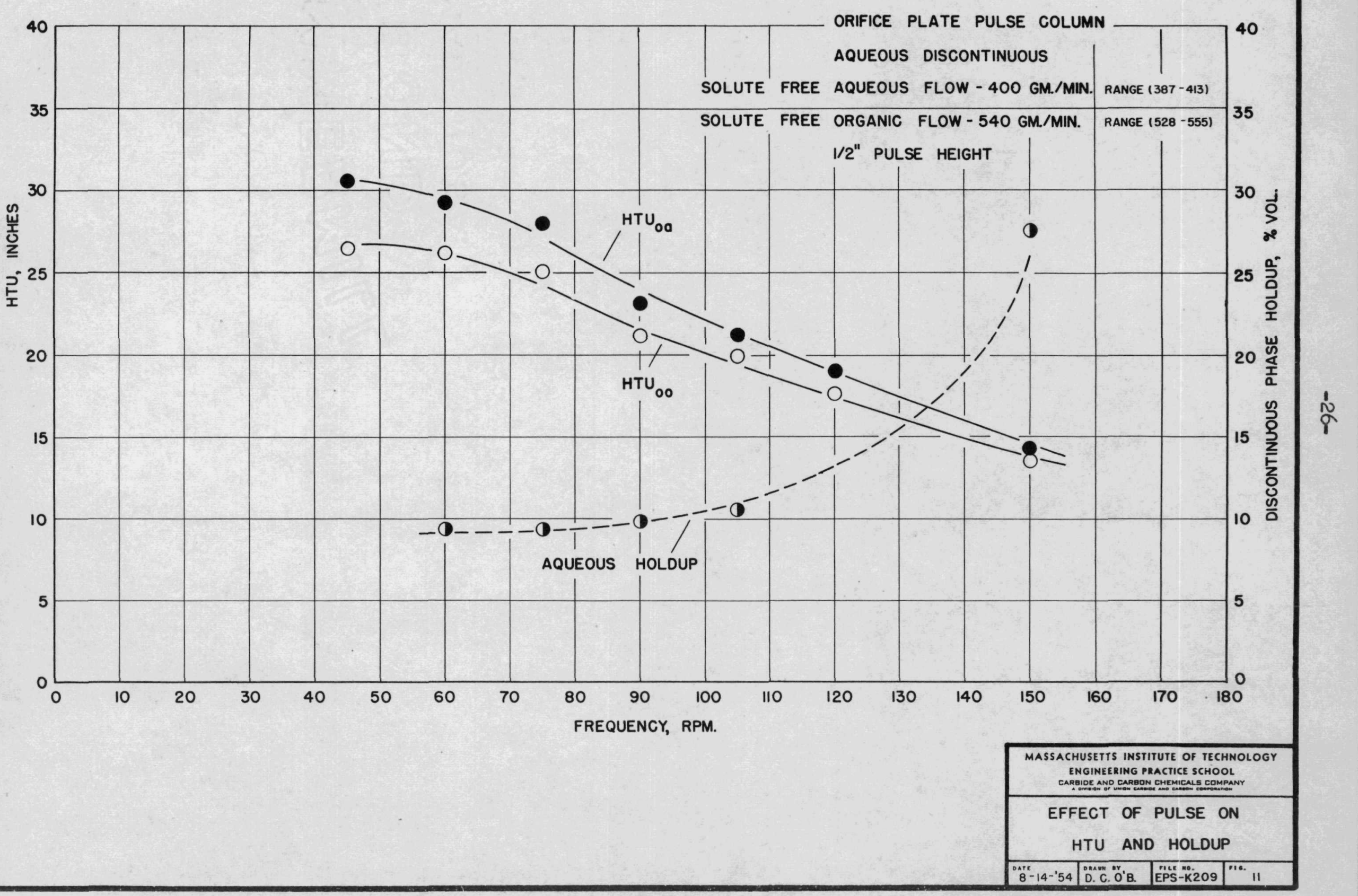




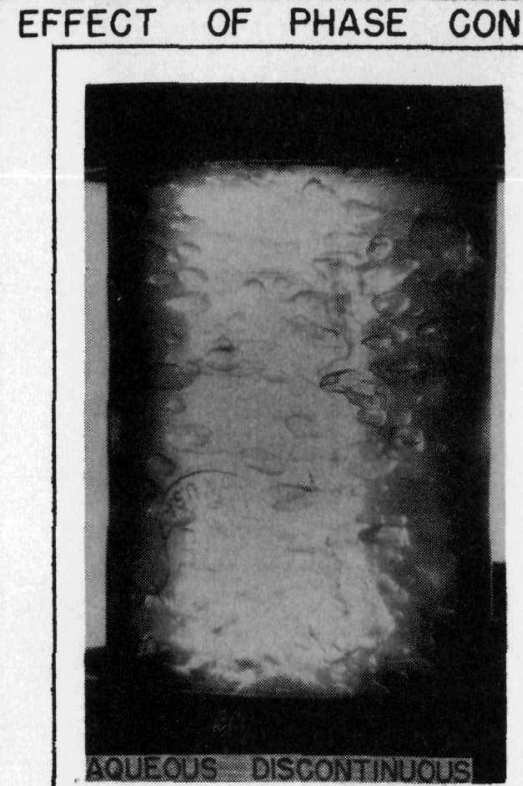

AQUEOUS FLOW- $240 \mathrm{GM} / \mathrm{MIN}$. TOTAL FLOW - $560 \mathrm{GM} . / \mathrm{MIN}$. FREQ. - 105 RPM., RUN 37 VOLUME HOLDUP- $7.2 \%$ HTU $_{00^{-18.2 " ~}}$ HTU $_{00^{-20.3 "}}$

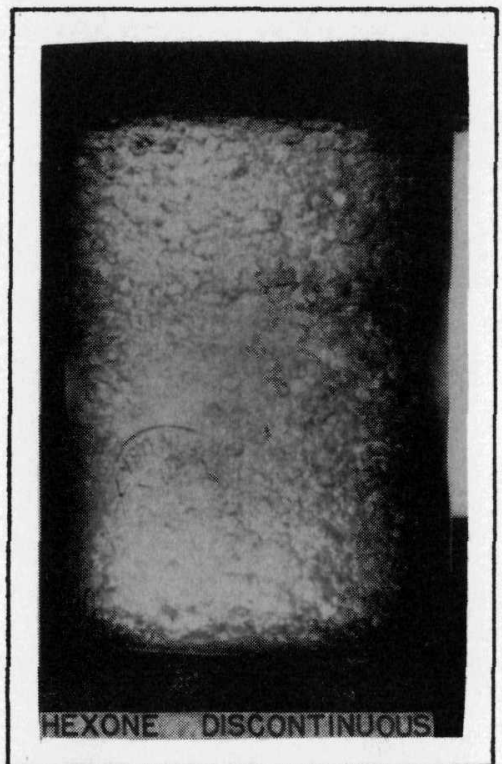

AQUEOUS FLOW-240 GM./MIN. TOTAL FLOW-560 GM./MIN. FREQ.- 105 RPM., RUN 69 VOLUME HOLDUP - $17.0 \%$ HTU $00^{-14.6^{\prime \prime}}$ HTU $_{\mathrm{Oa}^{-15.8}}$

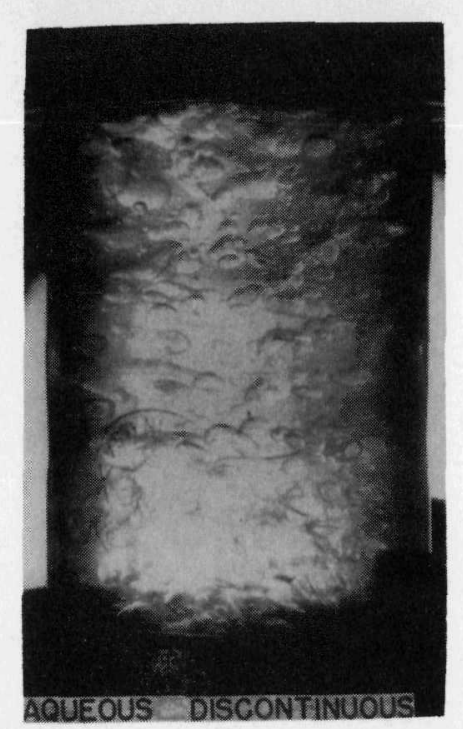

AQUEOUS FLOW -240 GM./MIN. TOTAL FLOW - 560 GM./MIN. FREQ. - 120 RPM., RUN 43 VOLUME HOLDUP- $7.4 \%$ HTU ${ }^{-16.9 "}$ HTU OO $^{-19.4 "}$

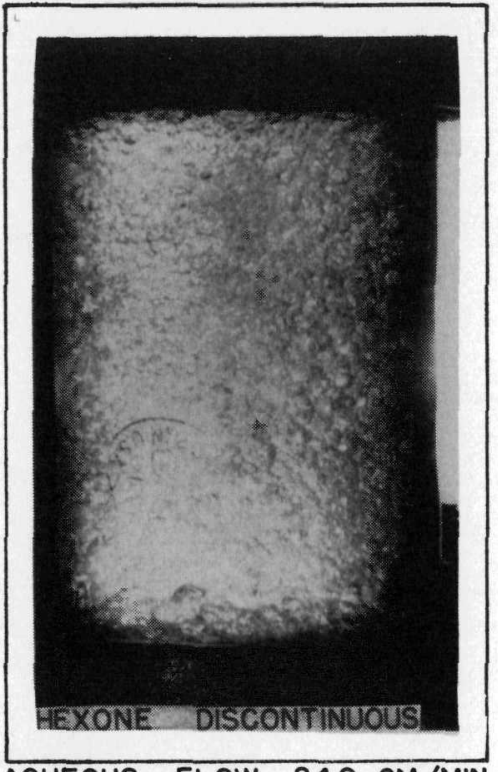

AQUEOUS FLOW-240 GM./MIN. TOTAL FLOW- 560 GM./MIN. FREQ.- 120 RPM., RUN 64 VOLUME HOLDUP- $22.3 \%$ HTU ${ }$-13.5" HTU $^{\prime \prime}$ Ha $^{-16.2^{\prime \prime}}$

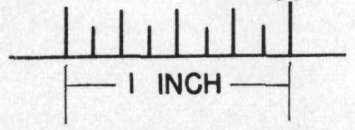

1/2" PULSE HEIGHT

FLOWS ON SOLUTE 'FREE BASIS

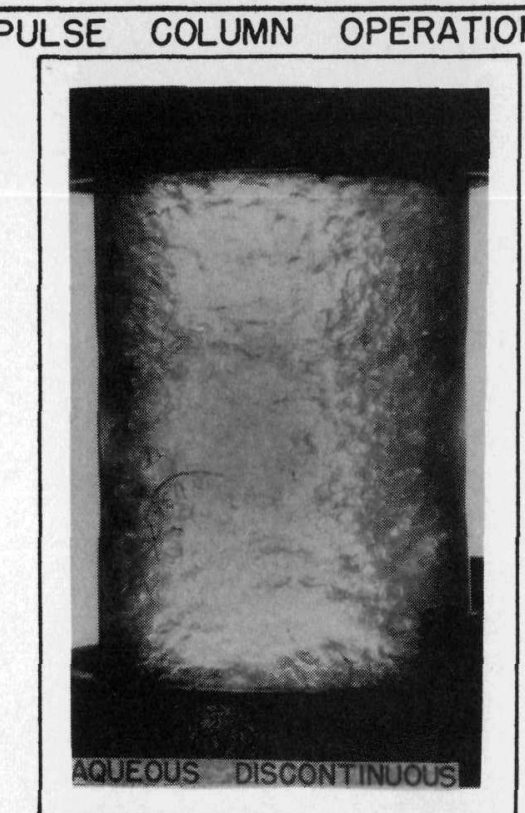

AQUEOUS FLOW- 240 GM./MIN TOTAL FLOW - 560 GM./MIN. FREQ.- 150 RPM., RUN 49 VOLUME HOLDUP- $14.5 \%$ $\mathrm{HTU}_{00}-14.2^{\prime \prime} \mathrm{HTU}_{\mathrm{OO}}{ }^{-16.1^{\prime \prime}}$

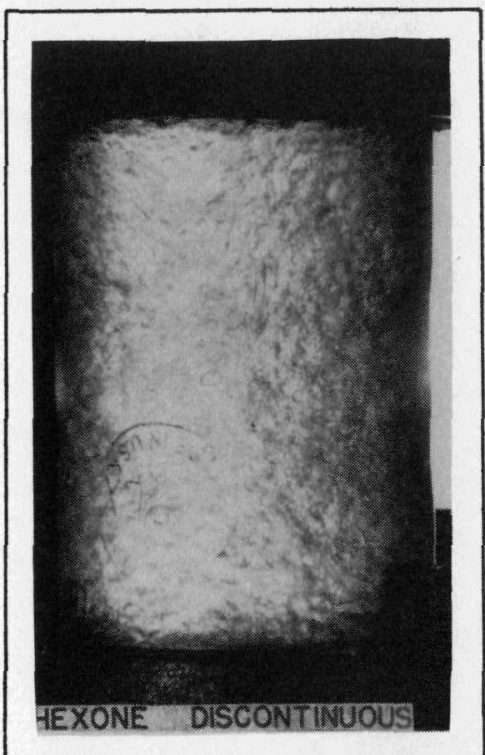

AQUEOUS FLOW- 240 GM./MIN. TOTAL FLOW - 560 GM./MIN. FREQ.- 150 RPM., RUN 70 VOLUME HOLDUP - $30.1 \%$ HTU OO- 14.1" HTU Oa- $15.3^{\prime \prime}$

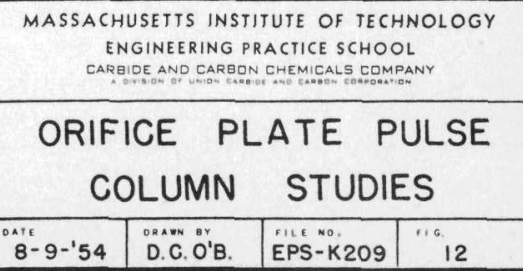


EFFECT OF THROUGHPUT ON ORIFICE PLATE PULSE COLUMN OPERATION

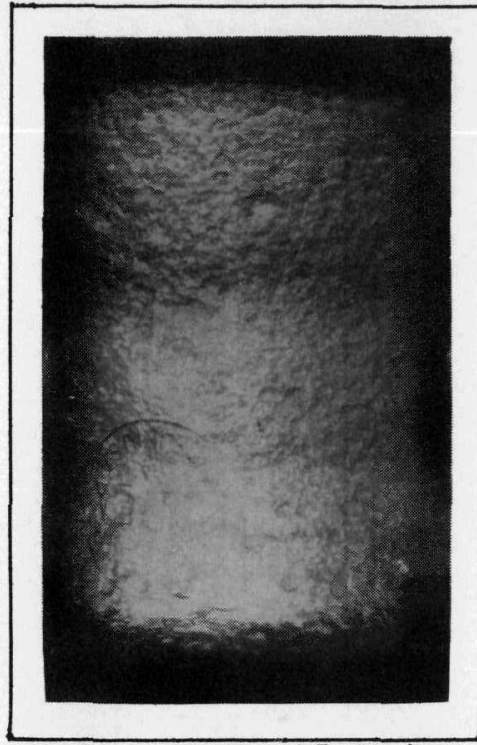

AQUEOUS FLOW - 75 GM./MIN.

TOTAL FLOW - 180 GM./MIN.

FREQ.- 120 RPM., RUN 41

VOLUME HOLDUP - $3.7 \%$

HTU ${ }_{O O}-23.9^{\prime \prime} \quad \mathrm{HTU}_{O \mathrm{O}^{-2}}{ }^{-26.7^{\prime \prime}}$

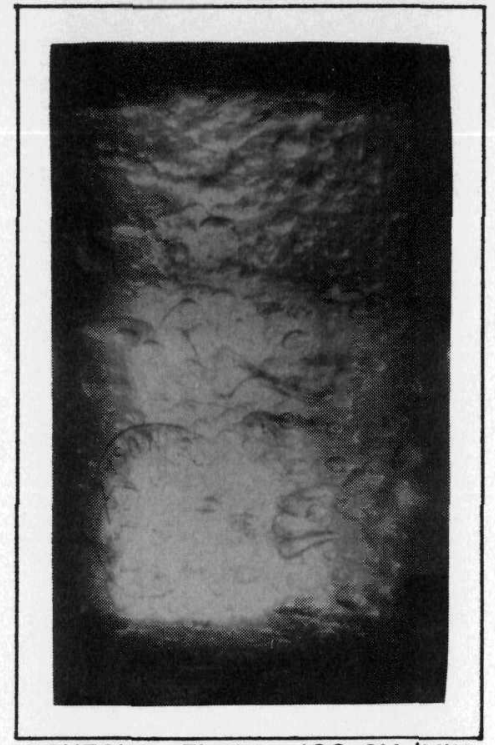

AQUEOUS FLOW - $160 \mathrm{GM} / \mathrm{MIN}$. TOTAL FLOW - 375 GM./MIN.

FREQ. - 120 RPM., RUN 42

VOLUME HOLDUP - $6.2 \%$

HTU $O O^{-17.7^{\prime \prime}}$ HTU $\mathrm{OQ}^{-20.5^{\prime \prime}}$

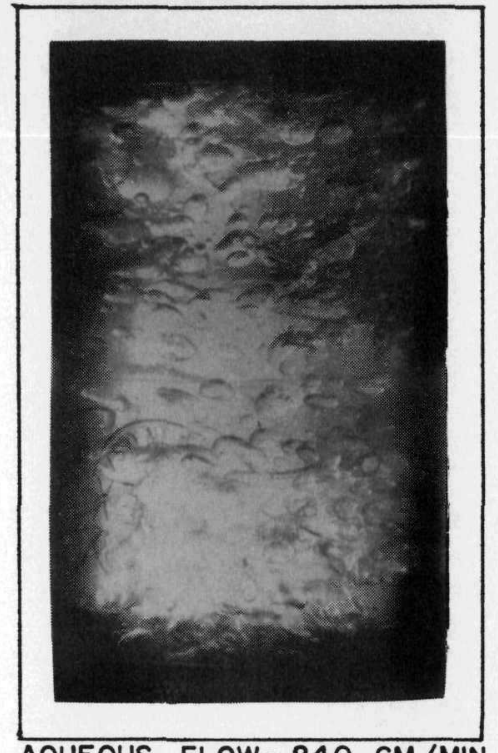

AQUEOUS FLOW- 240 GM./MIN TOTAL FLOW - $560 \mathrm{GM} . / \mathrm{MIN}$

FREQ.- 120 RPM. , RUN 43

VOLUME HOLDUP- $7.4 \%$

HTU $00^{-16.9^{\prime \prime} \quad \text { HTU }}{ }^{-19.4^{\prime \prime}}$

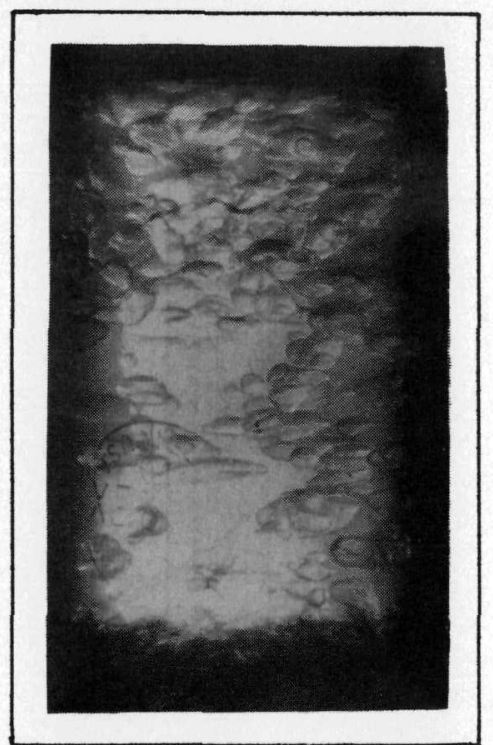

AQUEOUS FLOW- 320 GM./MIN. TOTAL FLOW - 740 GM./MIN. FREQ. - 120 RPM., RUN 44 VOLUME HOLDUP - $8.8 \%$ HTU $00^{-16.8 "}$ HTU OQ $^{-18.4^{\prime \prime}}$

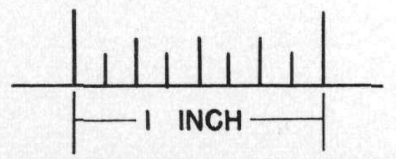

AQUEOUS DISCONTINUOUS I/2" PULSE HEIGHT FLOWS ON SOLUTE FREE BASIS

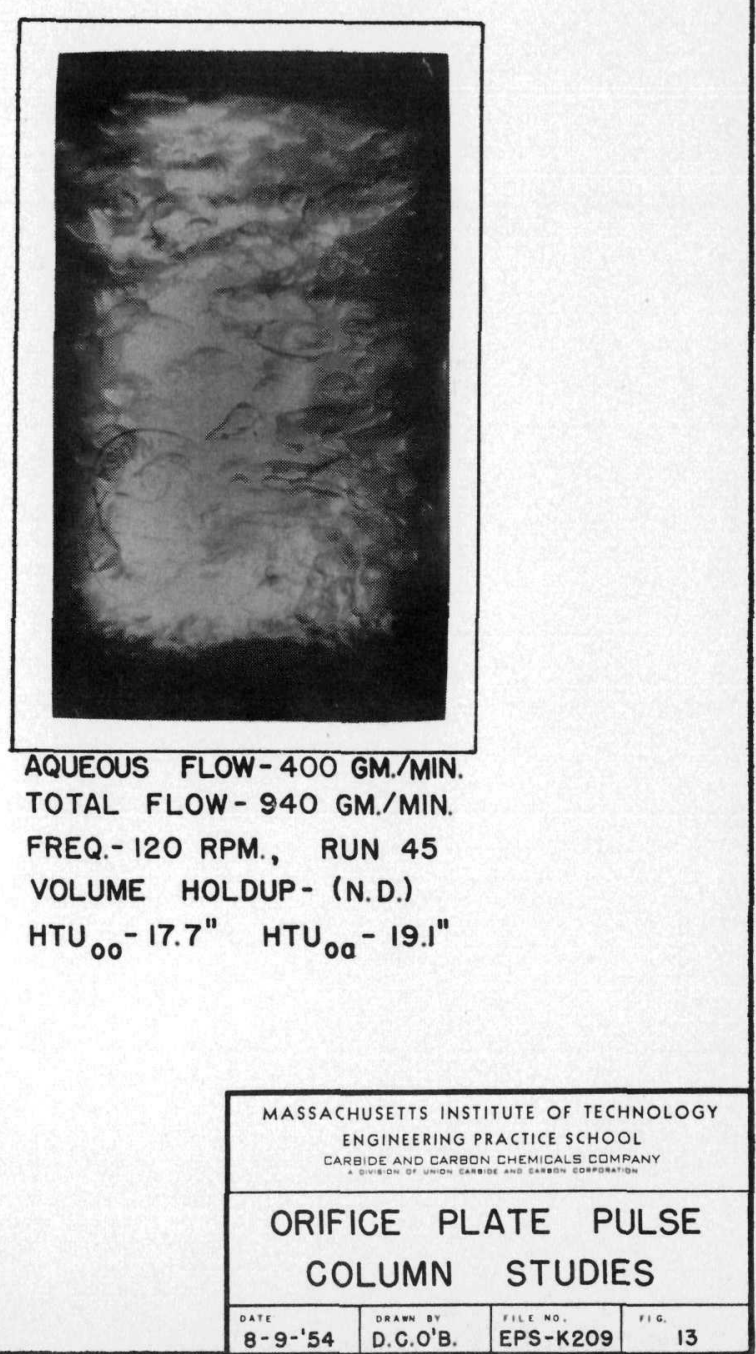


EFFECT OF THROUGHPUT ON ORIFICE PLATE PULSE COLUMN OPERATION

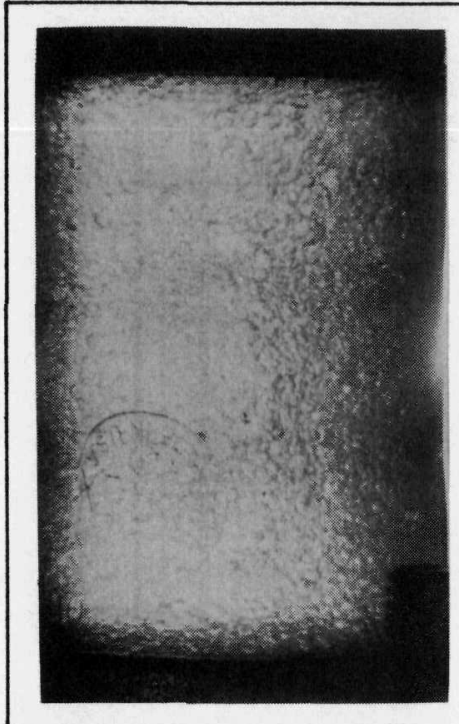

AQUEOUS FLOW- 75 GM./MIN. TOTAL FLOW - $180 \mathrm{GM} . / \mathrm{MIN}$. FREQ. - 120 RPM., RUN 62 VOLUME HOLDUP- $7.9 \%$ HTU $00^{-28.3^{\prime \prime}}$ HTU $_{O O^{-31.8 "}}$

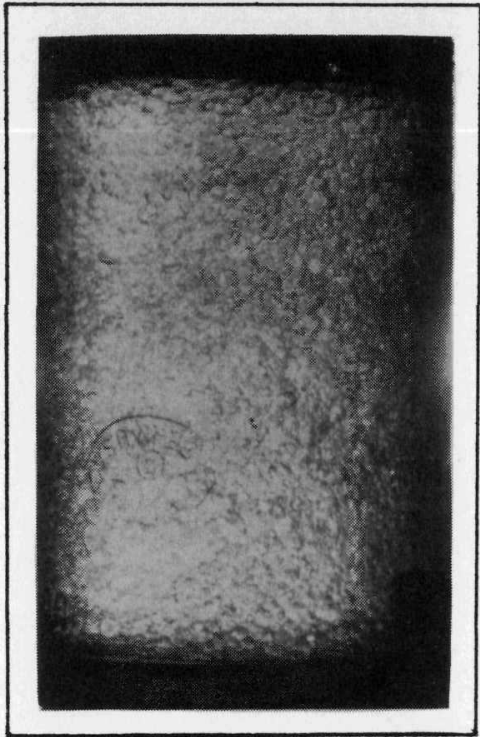

AQUEOUS FLOW- 160 GM./MIN. TOTAL FLOW- 375 GM./MIN. FREQ.- 120 RPM., RUN 63 VOLUME HOLDUP - $13.7 \%$ HTU $00^{-17.4 " ~ H T U ~}$ Ha $^{-20.3^{\prime \prime}}$

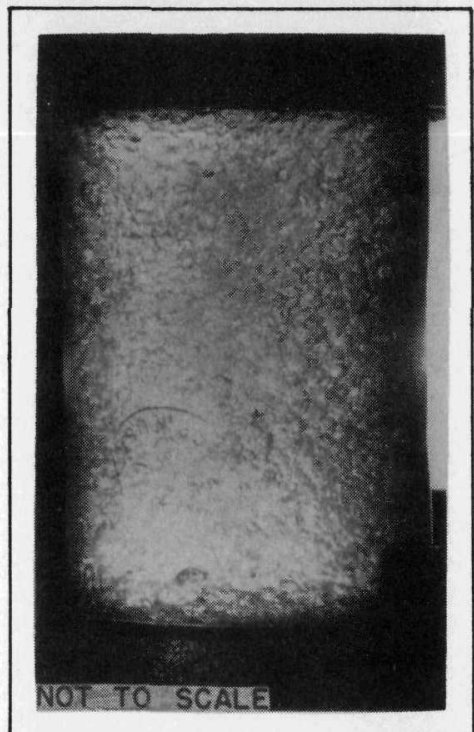

AQUEOUS FLOW- 240 GM./MIN. TOTAL FLOW-560 GM./MIN. FREQ. - 120 RPM., RUN 64 VOLUME HOLDUP- $22.3 \%$ HTU $00^{-13.5^{\prime \prime}}$ HTU ${ }^{\circ}{ }^{-16.2^{11}}$

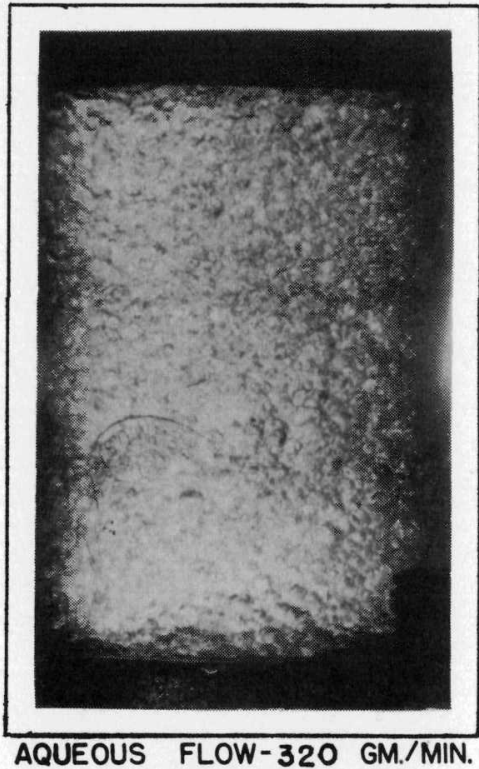

TOTAL FLOW-740GM./MIN.

FREQ.- 120 RPM., RUN 65

VOLUME HOLDUP- $29.4 \%$

HTU $00^{-13.8^{\prime \prime} \quad H T U_{00}-15.2^{\prime \prime}}$

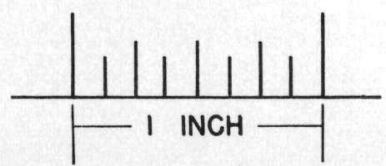

HEXONE DISCONTINUOUS

$1 / 2^{\prime \prime}$ PULSE HEIGHT

FLOWS ON SOLUTE FREE BASIS

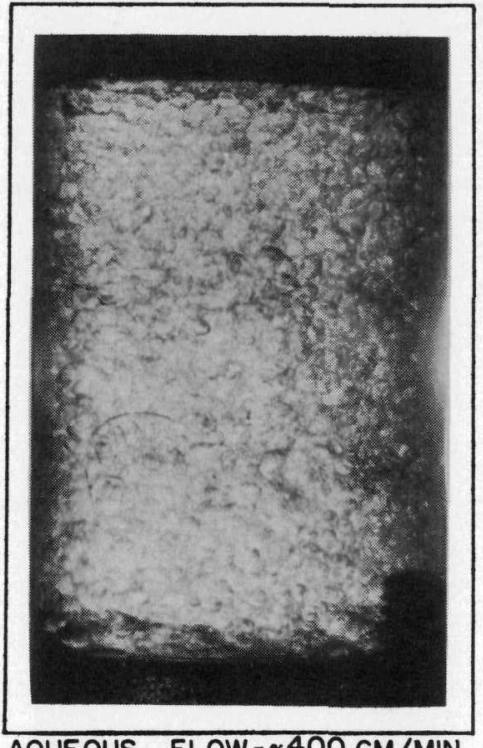

AQUEOUS FLOW $\sim 400 \mathrm{GM} . / \mathrm{MIN}$. TOTAL FLOW $\sim \sim 940 \mathrm{GM} / \mathrm{MIN}$. FREQ.- 120 RPM., NO RUN
MASSACHUSETTS INSTITUTE OF TECHNOLOGY ENGINEERING PRACTICE SCHOOL CARBIDE AND CARBDN CHEMICALS COMPAN

ORIFICE PLATE PULSE COLUMN STUDIES

\begin{tabular}{|c|c|c|c|}
\hline $8-9-$ & \begin{tabular}{|l|} 
ORANW \\
D.C.
\end{tabular} & $\begin{array}{l}\text { FILE } \\
\text { EPS }\end{array}$ & \\
\hline
\end{tabular}




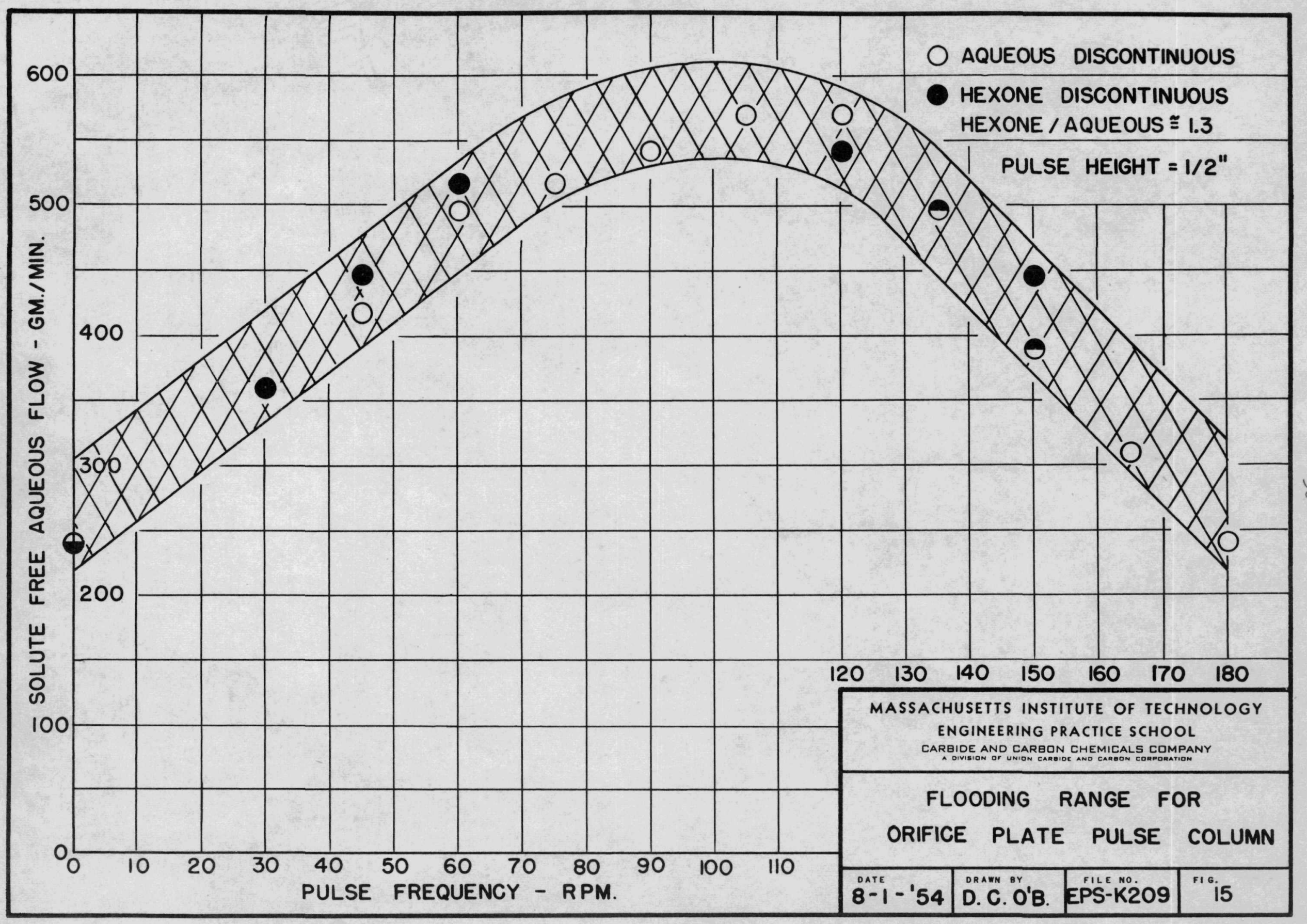




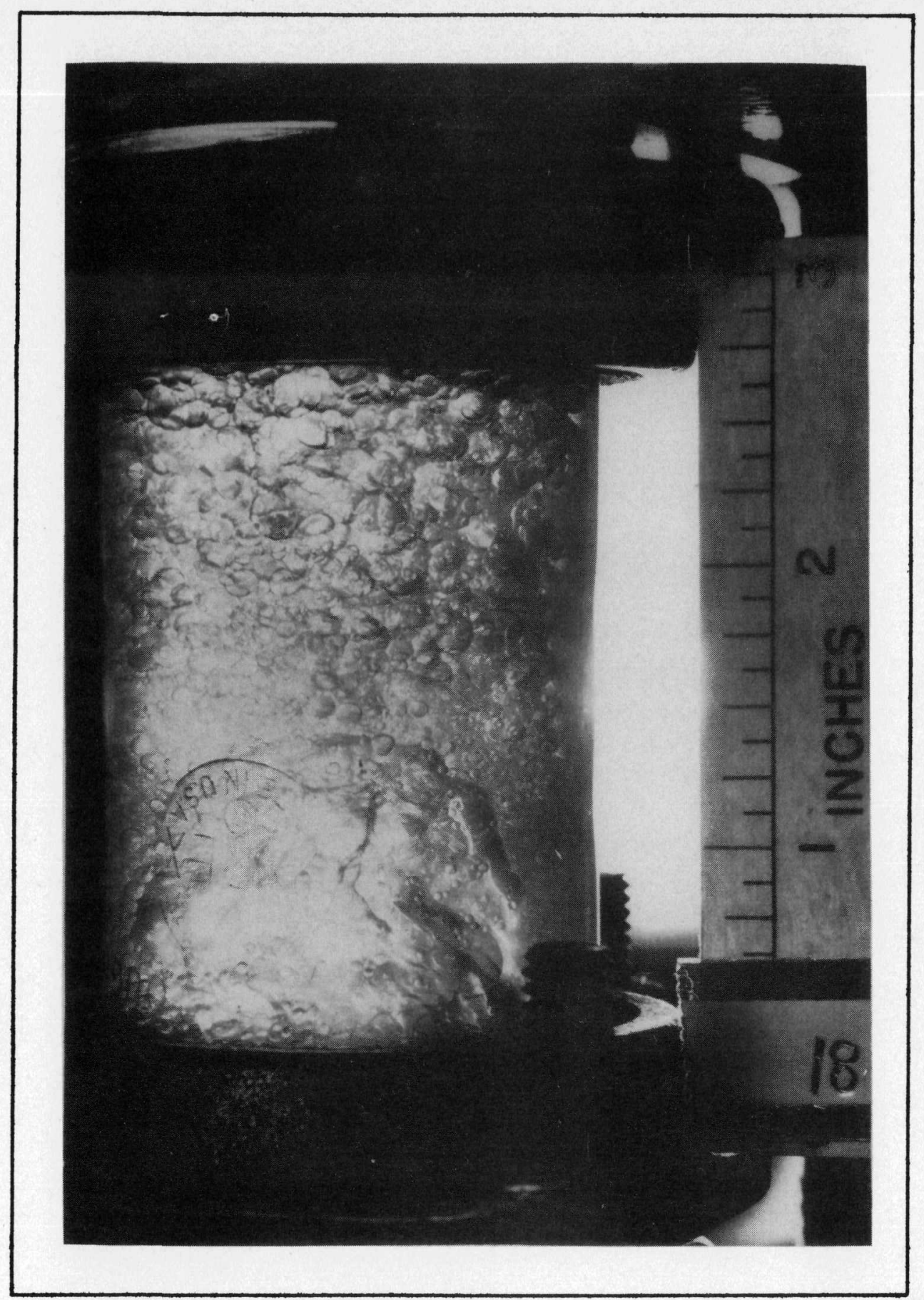

ORGANIC DISCONTINUOUS

MASSACHUSETTS INSTITUTE OF TECHNOLOGY ENGINEERING PRACTICE SCHOOL

CARBIDE AND CARBON CHEMICALS COMPANY

a division or union cakbioe ano carbon corporation

FLOODING IN AN ORIFICE PLATE PULSE COLUMN

\begin{tabular}{l|l|l|l}
\hline oATE & ORAEN BY & FILE NO. & IIG. \\
8-10-'54 & D.C. O'B. & EPS-K209 & 16 \\
\hline
\end{tabular}


EFFECT OF THROUGHPUT \& PULSE ON SPRAY TOWER OPERATION

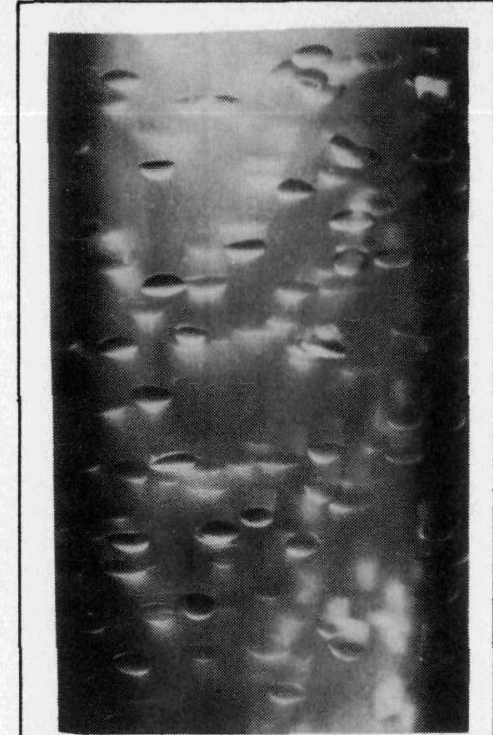

AQUEOUS FLOW - 75 GM./MIN. TOTAL FLOW - $180 \mathrm{GM} . / \mathrm{MIN}$. NO PULSE - RUN 75 VOLUME HOLDUP - $1.8 \%$ HTU $00^{-35.2^{\prime \prime}}$ HTU $00^{-399^{\prime \prime}}$

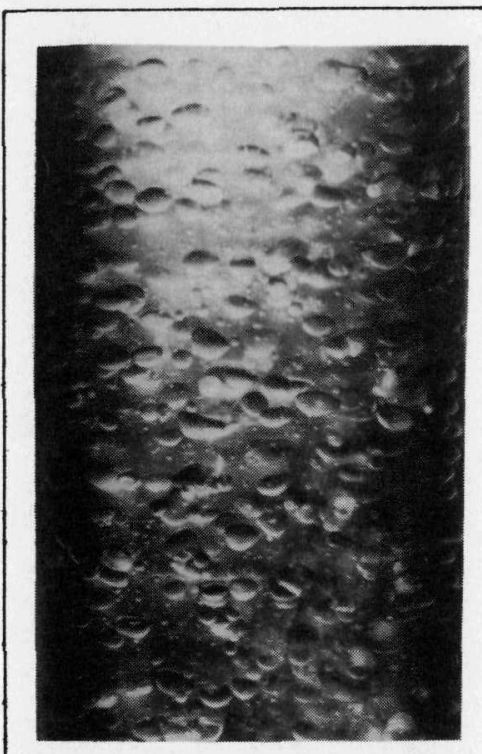

AQUEOUS FLOW- 225 GM./MIN. TOTAL FLOW - 545 GM./MIN. FREQ. - 200 RPM. - RUN 78 VOLUME HOLDUP - $10.8 \%$ HTU OO $^{-13.4^{\prime \prime}}$ HTU $_{0 a^{-14.2 "}}$

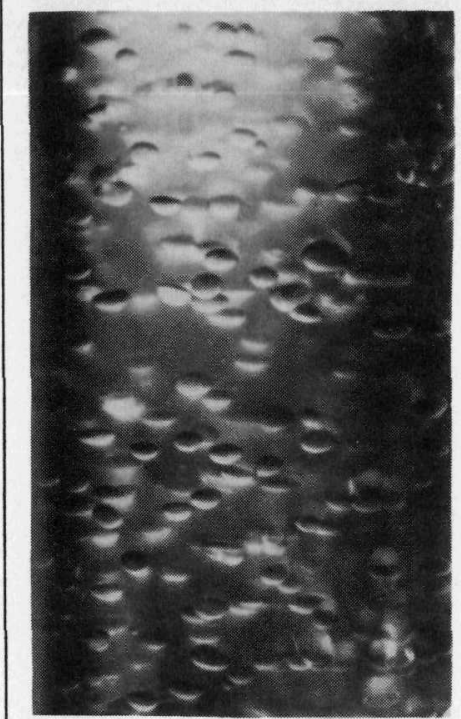

AQUEOUS FLOW - 150 GM./MIN. TOTAL FLOW-360 GM./MIN. NO PULSE - RUN 76

VOLUME HOLDUP $-5.9 \%$ HTU ${ }^{-17.9^{\prime \prime}}$ HTU Oa ${ }^{-19.5^{\prime \prime}}$

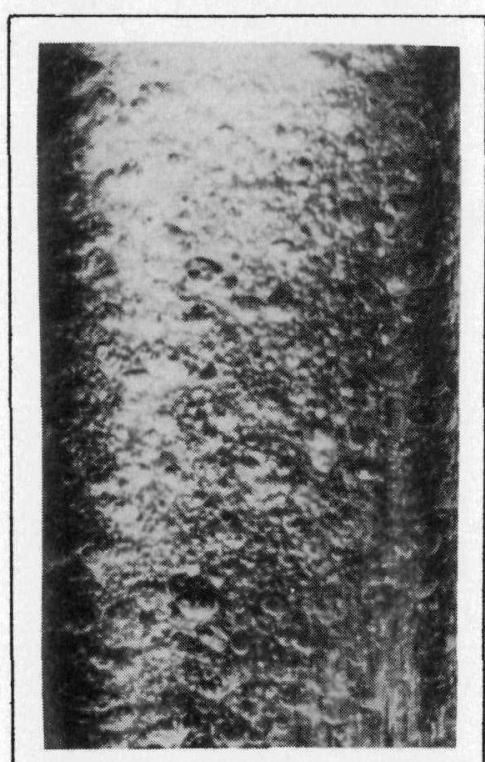

AQUEOUS FLOW-225 GM./MIN. TOTAL FLOW - 545 GM./MIN. FREQ. - 300 RPM. - RUN 79 VOLUME HOLDUP - $14.9 \%$ HTU $_{00}-11.4^{\prime \prime}$ HTU $\mathrm{Oa}^{-12.0^{\prime \prime}}$

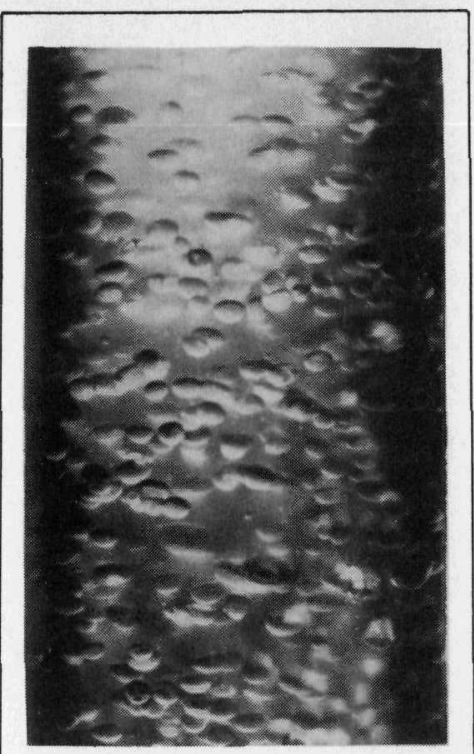

AQUEOUS FLOW- 225 GM./MIN. TOTAL FLOW-545 GM./MIN. NO PULSE - RUN 77 VOLUME HOLDUP-9.1\% HTU OO $^{-13.3^{\prime \prime}}$ HTU Oa ${ }^{-14.4^{\prime \prime}}$

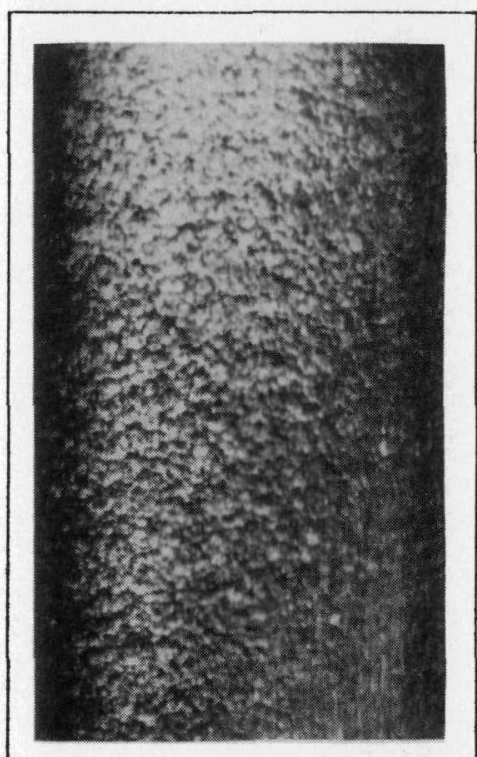

AQUEOUS FLOW- 225 GM./MIN. TOTAL FLOW - 545 GM./MIN. FREQ. -500 RPM.

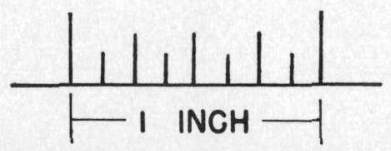

HEXONE DISCONTINUOUS

SPRAY TOWER STUDIES

I/2" PULSE HEIGHT

FLOWS ON SOLUTE FREE BASIS

8-6-'54 D.C.O'B. EPS-K209

17 


\section{TABLE I}

PRELIMINARY EXTRACTION RUNS (Aqueous Discontinuous)

\begin{tabular}{|c|c|c|c|c|c|c|c|}
\hline Run No. & $\begin{array}{l}\text { Frequency } \\
\text { RPM. }\end{array}$ & $\begin{array}{l}\mathrm{Q}_{\mathrm{a}} \\
\text { Solute Free } \\
\text { Aqueous Flow } \\
\text { gm./min. }\end{array}$ & $\begin{array}{l}{ }^{Q} O_{\text {Solute Free }} \\
\text { Organic Flow } \\
\text { gm./min. } \\
\end{array}$ & $Q_{0} / Q_{a}$ & $\begin{array}{l}\text { Per Cent } \\
\text { Deviation } \\
\text { Material } \\
\text { Balance } \\
\end{array}$ & $\begin{array}{l}\text { HTU } \\
\text { Inches }\end{array}$ & $\begin{array}{l}\text { HTU } \\
\text { Inchểs }\end{array}$ \\
\hline 1 & 30 & 83.0 & 160 & 1.93 & +0.8 & 45.9 & 33.9 \\
\hline 2 & 30 & 166 & 160 & 0.96 & -1.9 & 25.9 & 36.2 \\
\hline 3 & 30 & 332 & 160 & 0.48 & -0.2 & \multicolumn{2}{|c|}{--pinch-.- } \\
\hline 4 & 30 & 83.0 & 320 & 3.86 & -6.9 & 91.8 & 31.1 \\
\hline 6 & 30 & 165 & 317 & 1.92 & -0.3 & 45.4 & 32.9 \\
\hline 7 & 30 & 330 & 317 & 0.96 & -1.1 & 22.6 & 31.6 \\
\hline 8 & 30 & 74.2 & 63.2 & 0.85 & +0.8 & 27.2 & 63.2 \\
\hline 9 & 30 & 152 & 62.7 & 0.41 & +0.8 & \multicolumn{2}{|c|}{---pinch--- } \\
\hline
\end{tabular}


TABLE II

STRIPPING RUNS (Aqueous Discontinuous)

\begin{tabular}{|c|c|c|c|c|c|c|c|}
\hline Run No. & $\begin{array}{l}\text { Frequency } \\
\text { RPM. }\end{array}$ & $\begin{array}{l}Q_{a} \\
\text { Solute Free } \\
\text { Aqueous Flow } \\
\text { gm./min. }\end{array}$ & $\begin{array}{l}\text { Q } \\
\text { Solute Free } \\
\text { Organic Flow } \\
\text { gm./min. } \\
\end{array}$ & $Q_{0} / Q_{a}$ & $\begin{array}{l}\text { Per Cent } \\
\text { Deviation } \\
\text { Material } \\
\text { Balance } \\
\end{array}$ & $\begin{array}{l}\text { HTU } \\
\text { Inches }\end{array}$ & $\begin{array}{l}\text { HTU }_{\text {oa }} \\
\text { Inches }\end{array}$ \\
\hline 5 & 45 & 472 & 345 & 0.73 & -4.5 & 9.7 & 20.4 \\
\hline 10 & 75 & 730 & 535 & 0.72 & +7.8 & 9.6 & 17.5 \\
\hline 16 & 75 & 652 & 545 & 0.84 & +5.3 & 9.4 & 15.3 \\
\hline 22 & 60 & 627 & 574 & 0.92 & +3.2 & 12.6 & 19.2 \\
\hline 28 & 90 & 717 & 669 & 0.93 & +4.8 & 12.4 & 18.3 \\
\hline 34 & 105 & 626 & 567 & 0.90 & +4.5 & 9.9 & 17.9 \\
\hline 40 & 120 & 406 & 364 & 0.90 & +6.8 & 9.2 & 13.5 \\
\hline 46 & 120 & 518 & 470 & 0.91 & +4.3 & 11.5 & 16.4 \\
\hline 52 & 200 & 215 & 180 & 0.84 & +7.9 & 15.5 & 24.2 \\
\hline 58 & 105 & 629 & 556 & 0.88 & +2.4 & 12.0 & 18.7 \\
\hline 59 & 105 & 642 & 546 & 0.85 & +9.1 & 10.7 & 16.3 \\
\hline 66 & 120 & 518 & 461 & 0.89 & +8.5 & 9.5 & 13.7 \\
\hline
\end{tabular}




\section{DISCUSSION OF RESULTS}

A. Precision and Accuracy of Results

The calculations for this investigation were based on the assumption that the operating line was straight. Such an assumption was in error. In several of the preliminary extraction runs, inlet flow rates were measured with rotameters at the same time that outlet flow rates were determined by the "graduate and stopwatch" method. The latter method was used in nearly all of the runs. A comparison of the two measurements showed that the amount of water removed from the bottom of the column was less than the amount of water entering at the top. A similar comparison indicated more organic solvent leaving the column than had entered. From these observations, it was evident that some water was transferred to the organic phase.

These facts were consistent with a phase diagram used by Belaga and Bigelow (3) for the acetic acid, water, hexone system. Over the range of concentrations in the column the solubility of water in hexone practically doubled with the increase of acetic acid content in the hexone, whereas the solubility of hexone in water changed very little. Thus the use of mutually saturated feeds did not prevent the extraction of water into the hexone. The transfer observed was roughly $5 \%$ of the entering water. Measurements of solute free flow rates therefore were blased as evidenced by a consistently positive deviation in material balances (See Appendix, E. Summary of Data and Calculated Values). Moreover, the HIU values were also biased due to the change in flow rates along the column and the effect of the change on " $K$ ", the overall mass transfer coefficient, and "a", the transfer area per unit volume of column.

Another major source of error was the simplifying choice of a log mean driving force for the calculation of HTU. The equilibrium line is slightly curved (See Figure 20, Appendix). By graphical integration of a plot of concentration versus the reciprocal of the 
driving force, it was found that the HIU values calculated with the assumption were too high and thus conservative. The magnitude of the error increased as the HTU became smaller. The error was $11 \%$ for HTU values of 13 inches and 6\% for HTU values of 24 inches (See Appendix, D. Sample Calculations).

HTU values were also conservative from another standpoint. About 6 inches of the total 85 inches chosen as the column extraction length were not representative of true countercurrent flow. This 6 inches was the distance between the continuous phase inlet and the interface. No estimate was made of the portion of extraction which took place in this section. The interface was kept midway between the continuous phase inlet and the discontinuous phase outlet.

Accuracy of the equilibrium curve also constituted a source of error. The data used (See Appendix, B. Chemical System and Analytical Methods) were for a temperature range of $82-90 \mathrm{~F}$. and were obtained from Belaga and Bigelow (2). According to these investigators, the data were substantially in agreement with Sherwood, Bvans and Longcor (Ind. Eng. Chem., 31, 1144 (1939)) but not with Othmer, White, and Trueger (Ind. Eng. Chem., 33, 1240, (1941)). Room temperatures were recorded in this study as an indication of tower temperatures and varied from 75 to 95 OF. A comparison of equilibrium data by Fleming and Johnson (5) taken over a range of 69 - 75 OF. With those of Belaga and Bigelow indicates that the error due to change in equilibrium with temperature is probably slight. Since the equilibrium curve was fixed, the lower HIU values had more per cent error due to smaller driving forces.

Normalities were reported to 4 significant figures with a precision of $0.5 \%$. Concentrations of samples taken from the same outlet stream as an indication of steady state operation usually checked within $1-2 \%$. In several cases the first sample disagreed with the second by as much as $10 \%$. The majority of these samples were of the aqueous phase taken from aqueous discontinuous runs at low throughput. Material balances for these runs usually agreed more closely when concentrations of the second samples were used. 
At steady state operating conditions, checks on volume flow measurements were found to vary by as much as $3 \%$. The combined error of the graduate and stopwatch was estimated to be $0.5 \%$. The remaining error was attributed to fluctuations in inlet flows, interface level variations, slugging in the outlet lines and imprecisions in the measuring techniques. Hydrometers were calibrated to 1 part in the third decimal place. Errors in specific gravity measurements due to temperature variations were estimated to be somewhat larger than reading errors of the hydrometers, but were less than $1 \%$. Pulse height was measured to the mearest $1 / 32$ inch and error in the frequency measurement was negligible.

Holdup data probably were no more accurate than 10\%. Measuring techniques probably contributed most to the scatter of the data. Quick closing valves were not used, thus making it difficult to shut off flows quickly and simultaneously. Some error was undoubtedly due to actual operating fluctuations.

Flooding data similarly do not have a high degree of accuracy, primarily because the degree of coalescence was somewhat arbitrary and flooding was a transient phenomenon. Error in flooding determination also was partly due to the use of discreet values of throughput rather than a continuous spectrum. Quantitative application of the flooding data to other columns is not advisable since the effect of the inlet constrictions at the distributor nozzles was not known.

B. Factors Affecting HTU

The following relationships were used in expressing extraction efficiencies based on the organic phase: (Similar equations were used for calculations based on the aqueous phase.)

$$
\begin{aligned}
& \mathrm{NIU} \mathrm{O}_{00}=\int_{Y_{1}}^{Y_{2}} \frac{d Y}{\left(Y^{*}-Y\right)}=\int_{0}^{Z} \frac{K a S d z}{Q_{0}} \\
& \mathrm{HTU}_{\mathrm{OO}}=\mathrm{Z} /_{\text {NTU }}
\end{aligned}
$$




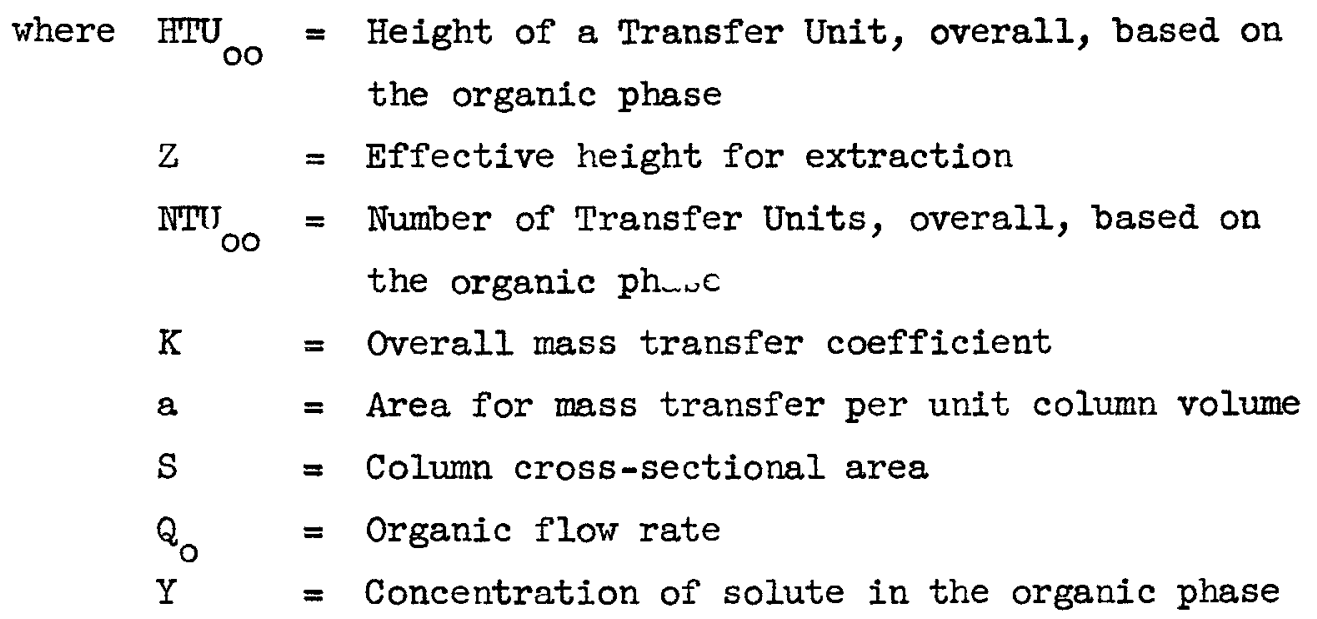

Analysis of the effect of a variable on extraction efficiency thus requires an examination of the effect of that variable on " $K$ ", " $a$ ", and the validity of the assumption that the operation truly can be represented by a differential equation.

The validity of the assumption of differential operation is reduced by stage-wise operation. Moreover, representation by differentials would not present a true picture if the column operated with back-mixing, a phenomenon which effectively reduces the number of countercurrent stages available for extraction. Back-mixing is essentially a localized reversal of countercurrent flow to cocurrent flow which tends to mix higher concentrations of a phase with lower concentrations of that phase. Since back-mixing would therefore tend to lower the driving force for mass transfer throughout the column, it would consequently tend to increase the HIU.

True spray column operation may be considered as differential, whereas studies by Gavin and Thies (6) show that a sieve plate pulse column essentially operates as a stage-wise device. Idealized operation of the columns is based on the assumption that little or no back-mixing occurs in a non-pulsed spray column or in a sieve plate pulse column, because the plates allow phase separation at each stage. Back-mixing would be much more probable in an orifice plate pulse column than in a sieve plate pulse column due to the lower resistance to flow of the orifice. 
As the frequency of pulsing is increased, the acceleration of the fluids is also increased, tending to increase the shear forces on the discontinuous phase at the walls and the orifice plates. It would seem logical, therefore, that average arop size would decrease with increased frequency. Visual observations showed that drop size did vary inversely with frequency (See Figures 8, 9). Stoke's Law predicts that small drops move more slowly through the continuous phase than large drops because they have more surface resistance per unit mass. A decrease in drop size of the discontinuous phase at constant throughput would mean an increase in the discontinuous phase holdup. The results are in accord with this reasoning and show an increase in holdup with pulsing, particularly at higher frequencies (See Figures 5-11). The combination of smaller arops and increased holdup, with the related possibility that small, slowmoving drops would be carried cocurrently with the continuous phase, suggests that back-mixing would increase with frequency.

As the flooding range was approached, agglomeration and coalescence of the drops occurred as a result of increased holdup. Although coalescence would tend to increase the average drop size, it would also tend to reduce back-mixing to some extent. Current theories suggest that coalescence followed by regeneration of surface area tends to increase transfer and lower the HIU (ㄹ, 12). At very low frequencies coalescence occurred due to a longer settling time afforded the larger drops on the reverse pulse stroke (See Figures 8, 9).

The following is a list of factors influencing HIU which are affected by pulsing:

Effect of Increased Pulsing Below the Flooding Range

\begin{tabular}{|c|c|c|c|c|}
\hline Factor & $\begin{array}{l}\text { Change in } \\
\text { Factor }\end{array}$ & $\begin{array}{l}\text { Independent } \\
\text { Change in "a" }\end{array}$ & $\begin{array}{l}\text { Independent } \\
\text { Change in " } \mathrm{K}^{\text {" }}\end{array}$ & $\begin{array}{l}\text { Independent } \\
\text { Change in HTU }\end{array}$ \\
\hline drop size & decrease & increase & --- & decrease \\
\hline $\begin{array}{l}\text { terminal drop } \\
\text { velocity }\end{array}$ & decrease & --- & decrease & increase \\
\hline holdup & increase & increase & $-\cdots$ & decrease \\
\hline back-mixing & increase & --- & --- & increase \\
\hline $\begin{array}{l}\text { turbulence and } \\
\text { drop oscillation }\end{array}$ & increase & $-\cdots$ & increase & decrease \\
\hline $\begin{array}{l}\text { coalescence and } \\
\text { regeneration of } \\
\text { surface area }\end{array}$ & decrease & 240 & decrease & increase \\
\hline
\end{tabular}


Several investigators have found "Ka" for studies on spray and packed columns to vary almost linearly with the discontinuous phase flow rate up to the flooding point (10). The effect of the continuous phase flow rate on "Ka" is small but not negligible (10). Assuming that the distributor produced drops of approximately the same size over the range of throughputs investigated, any change in drop size with increased throughput would be due primarily to increased coalescence. Lurie and Shaver (I) found that the average drop size in their orifice plate column increased with an increase in the dispersed phase flow rate. Photographs for this investigation (See Figures 13 and 14) were consistent with their observations. Since the pictures and the data showed a considerable increase in holdup with throughput, it was concluded that increased throughput affected "Ka" primarily by increasing "a", regardless of the increase in drop size. An increase in drop size, however, would tend to increase terminal velocity by Stoke's Law (10) and thus to raise " $K$ ". Moreover, increased turbulence and drop oscillation would also tend to - raise " $K$ ". Since the drop size did change appreciably with throughput, especially when the aqueous phase was dispersed, it is believed that back-mixing decreased with increasing throughput.

The following is a list of factors influencing HIU which are affected by throughput:

Effect of Increased Throughput Below the Flooding Range

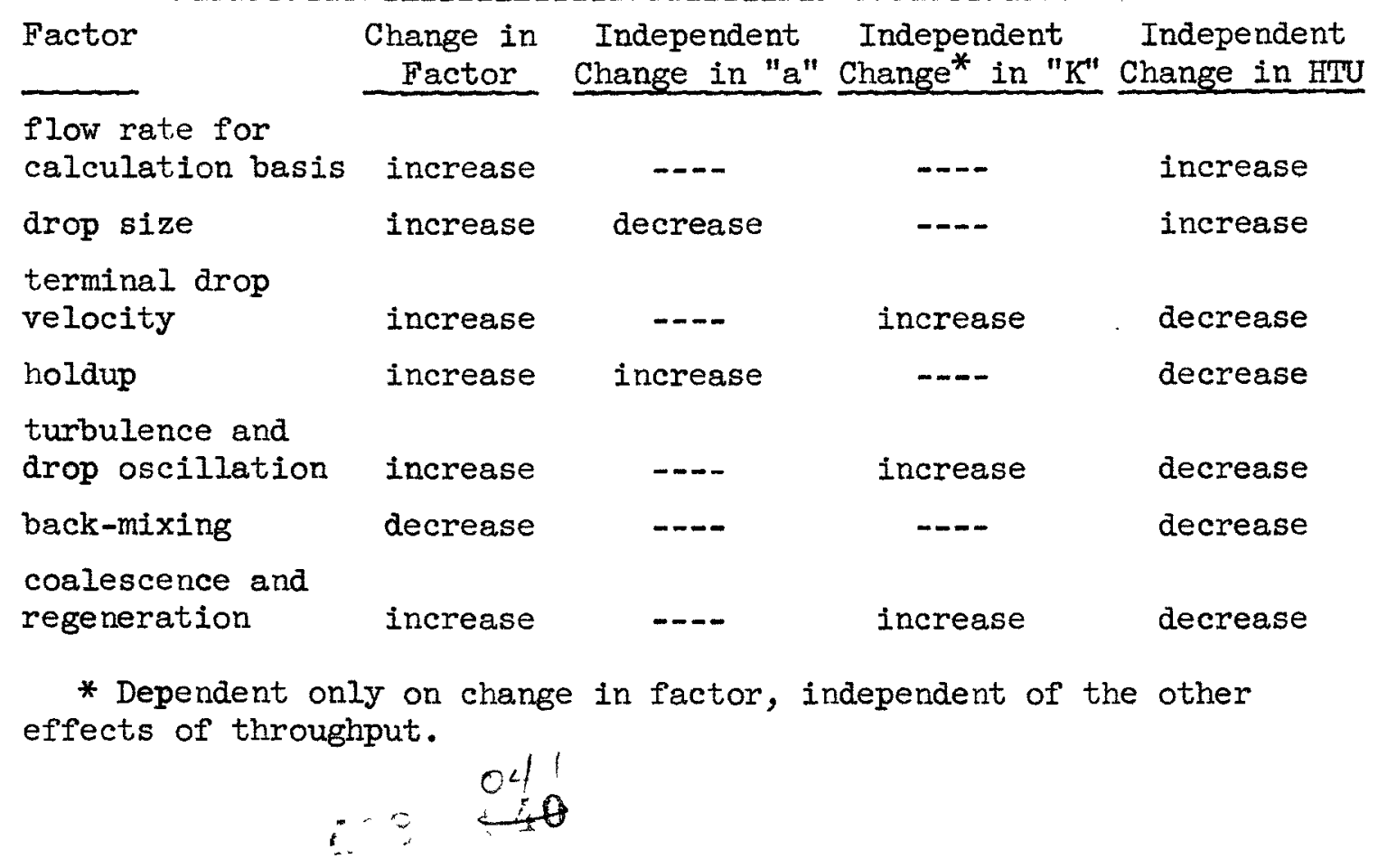


Choice of a particular phase to be discontinuous is dependent upon at least three factors. Normally the phase receiving solute is dispersed in order to reduce coalescence (5, 10). In order to increase surface area, the phase which preferentially wets the packing (or orifice material) should be dispersed (10). For the same reason, the phase with the larger volume flow rate should be dispersed. For the chemical system and the flow ratio used in this - investigation, these factors all favor dispersion of the organic phase in extraction runs.

\section{Examination of HTU Results}

Both HIU 00 and HIU were calculated for this investigation.

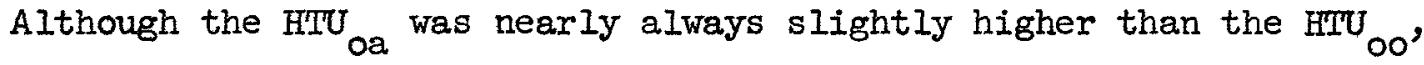
the following discussion uses HIU in reference to both since their curves showed the same characteristics.

1) Aqueous Phase Dispersed - Transfer into Organic Phase

As may be seen in Figure 5 (75 gm./min. solute free aqueous flow), the HIU changed only slightly over the range of $0-45$ RPM. The change, if any, was probably due to a decrease in transfer area by coalescence (See Figures 8 and 9), since the column flooded easier at lower frequencies than at intermediate frequencies (See Part $D$, Flooding Range Curve). Holdup also changed very little over this range. The HTU decreased slowly and steadily from 45-105 RPM; the holdup increased roughly in proportion. The decrease of the HTU over this range probably could be attributed to a controlling effect of increased transfer area and turbulence. After $105 \mathrm{RPM}$, the HTU slowly increased. Although holdup increased at a faster rate than at lower frequencies, apparently the increase in transfer area was overbalanced by increased back-mixing.

The curves in Figure 6 (160 gm./min. solute free aqueous flow) exhibit much the same properties as those in Figure 5. The minimum HIU obtained at these conditions was somewhat lower than for the smallest throughput, however, and relatively little increase was noted in the HIU after 120 RPM. Holdup again increased faster at 
higher frequencies than at lower frequencies. Apparently, for this throughput, back-mixing in the orifice plate column was still a controlling factor at high holdups.

For the orifice plate pulse column, change in throughput below the flooding range produced little change in the HTU at low frequencies. Preliminary Runs $I$ and 6 shown in Table $I$ indicated this fact as did the curves in Figures 5-7, 10 and 11. At higher frequencies, however, an increase in throughput decreased the HIU as was discussed in Part B. Since holdup inereased with throughput in all cases, it was concluded that the decrease in HIJ observed for all curves as a function of throughput was due primarily to increased transfer area, although increased turbulence, increased drop oscillation and decreased backmixing definitely were influencing factors.

Referring to Figure 7 (240 gm./min. solute free aqueous flow), data were not taken at $O$ and 180 RPM because the column flooded at those frequencies. Over the frequency range of 45-165 RPM., the HTU decreased steadily and more rapidly than was observed at lower throughputs. Holdup again increased over this range, more noticeably at higher frequencies, and apparently back-mixing was not a controlling factor when throughputs were also high.

The curves in Figures 10 and 11 (320 and $400 \mathrm{gm} . / \mathrm{min}$. solute free aqueous flow) exhibit much the same properties as the curves in Figure 7, except that the HTU values were respectively lower and the holdup values were higher. As was mentioned earlier, these differences were attributable to increased transfer area, increased turbulence and drop oscillation, and decreased back-mixing as a result of increased throughput.

\section{2) Organic Phase Dispersed - Transfer into Organic Phase}

The HTU (organic discontinuous) curve on Figure 7 exhibits a minimum over a frequency range of 45-165 RPM., but the overall change in HIU was relatively slight. Although holdup increased with frequency, apparently the factors affecting HIU were compensating. At this throughput, the HTU was lower for organic discontinuous than for

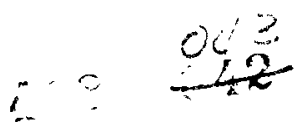


aqueous discontinuous. This observation may be explained in part by the fact that the discontinuous phase holdup, and thus transfer area, was higher when the organic phase was dispersed (See Figure 12).

Examination of Figures 5-7, 10 and 11 at 120 RPM shows that the organic discontinuous holdup was always higher, and yet at low throughputs the organic discontinuous HIU was higher or equal to the aqueous discontinuous HTU. It was therefore concluded, as in the case of aqueous discontinuous, that back-mixing was an important factor at high holdups and low throughputs.

D. Flooding Range Curve (Figure 15)

The most important point for discussion is the fact that the flooding range curve for the orifice plate pulse column exhibits a maximum. Apparently at low frequencies, pulsing tends to increase the maximum throughput by pumping material from one cell to the next. Visual observations of this pumping action have been noted in other investigations (2, 3 , 12), especially for sieve plate pulse columns. For idealized operation of a sieve plate pulse column, the maximum throughput would be equal to twice the pulse displacement times the frequency, if no back-mixing occurred.

As was previously discussed, however, holdup and thus backmixing did increase with increased frequency. Moreover, it was mentioned that coalescence also was more probable at higher holdups. All these factors would tend to decrease the maximum throughput. It is reasonable, therefore, to expect that at some frequency, holdup and back-mixing would overbalance the effect of pumping action and decrease the maximum throughput with increased frequency thereafter.

Buchanan, et. al. (3) found that flooding data for a sieve plate pulse column could be correlated with frequency when flow was expressed as the sum of the flows of both phases. Although the same phase was always dispersed, the correlation held for several phase ratios. Other investigators (5, 10) have found that flooding is dependent upon both flow rates, one varying inversely with the other at maximum throughput. Since the phase ratio was approximately 
constant for this investigation, it is easily seen that the flooding data could have been correlated as well with total flow as with the flow of either phase.

An interesting point for discussion is the fact that data from organic discontinuous studies as well as aqueous discontinuous studies were used in obtaining the flooding range curve. No detailed analysis was made of this observation, but it appears that for this system, flooding was probably more dependent upon total flow than upon flow of either discontinuous phase.

\section{E. Discussion of Overall Operation}

For the conditions of this investigation, the orifice plate pulse column produced minimum HIU values very near to 1 foot. These values were obtained at high frequencies and high flow rates, very close to flooding conditions. For the same chemical system, the same phase dispersed, and the same direction of transfer, at a similar flow ratio, but much lower flow rates, Belaga and Bigelow (ㄹ) obtained HTU values as low as 3 inches in a sieve plate pulse column. In general, they found extraction to be best at low frequencies and low pulse heights. Their column had the same internal diameter as the orifice plate pulse column, and the plates had very nearly the same free area, but their plate spacing was 1 inch and pulse heights ranged from $1 / 8$ to 2 inches. Later work on the same sieve plate pulse column by Baker and Lang (1) showed that HIU values were lowest when the column was operated almost at flooding. In their investigation, however, frequency was kept constant at $40 \mathrm{CPM}$. and no flooding data as a function of frequency were obtained for comparison with results of the current investigation.

Quantitative comparison of the two types of extraction devices therefore requires some speculation. Certainly there is no indication that the design of the orifice plate pulse column used in this investigation was optimum from the standpoint of either throughput or extraction. Since Gavin and Thies (6) found that extraction in Belaga's column varied inversely with the distance between plates, over a range 
of plate spacing of $1 / 4$ to 2 inches, probably reducing the plate spacing on the orifice plate pulse column would also improve its extraction characteristics. Moreover, the current investigation provided little knowledge of the effect on extraction of orifice size and pulse amplitude, undoubtedly two very important variables.

The most interesting comparison was made by Buchanan, et. al. (3) for another chemical system, using larger equipment than was used for this investigation. In their investigation an orifice plate pulse column achieved the same extraction as was obtained from a sieve plate pulse column operating at the same flow rates. At comparable frequencies they also found that the orifice plate pulse column permitted a higher throughput.

Based on development up to this time, it is felt that the orifice plate pulse column has less favorable extraction characteristics than the sieve plate pulse column, but permits a higher maximum throughput. Therefore, just as the spray tower is frequently chosen in preference to the packed tower because of its higher throughput, although its extraction may not be as favorable (10), situations probably exist where the orifice plate pulse column would be more desirable from economic considerations than the sieve plate pulse column. Certainly the possible use of orifice plate pulse columns in slurry extraction is a potentially important application.

A comparison of pulsed and nonpulsed spray tower operation (Seè Figure 17) with that of the orifice plate pulse column also requires considerable speculation. As was expected, the three organic discontinuous nonpulsed spray tower runs showed a fairly large decrease in HWU with increased throughput. At $240 \mathrm{gm} . / \mathrm{min}$. solute free aqueous flow, the HTU value obtained for spray tower operation was 14 inches, a value equal to the lowest HIU achieved by the orifice plate column at the same throughput over the frequency range studied.

At the same throughput, pulsing the spray tower at 200 RPM. resulted in no change in HTU although the holdup increased slightly. 
However, when the spray tower was pulsed at 300 RPM., again at the same throughput, the HIU decreased to 11 inches, the lowest obtained throughout the investigation. It is suspected that the increased dispersion and holdup observed for this latter run were not independent of the type of distribution plates employed and the resulting constrictions at the inlets (See Figure 18). The results, however, were in direct contrast with the observations of Lurie and Shaver (7). Although these results are not conclusive, it would appear that pulsing a spray tower may increase extraction efficiency. The spray tower is a limiting design of an orifice plate column; i.e., with the orifice diameter equal to the tower diameter. Under these conditions throughput would be expected to be a maximum, thus increasing the potential of the pulsed apparatus as an extraction device. 


\section{CONCLUSIONS}

On the basis of the results of this investigation it was concluded that:

1) Pulsing tended to increase holdup and improve extraction efficiency, particularly at high frequencies and at high throughputs.

2) Pulsing tended to create smaller drops and increase backmixing, particularly at low throughputs.

3) An increase in throughput tended to increase holdup and improve extraction efficiency, particularly at high frequencies. $\because \quad 4)$ An increase in throughput tended to increase drop size and decrease back-mixing.

5) Dispersion of the organic phase in preference to the aqueous phase, when solute transfer is into the organic phase, resulted in more holdup and increased transfer area. These factors increased the extraction efficiency at high flow rates and low frequencies.

6) Pulsing in an orifice plate pulse column tended to increase maximum throughput by pumping action and to decrease maximum throughput by increasing holdup. Maximum throughput was highest in the range of intermediate frequencies. 


\section{RECOMMENDATIONS}

In view of the effects of pulsing in an orifice plate pulse column and the possibility that the potential of the spray tower as an extraction device may be increased by pulsing, it is recommended that:

1) A more detailed study be made on an orifice plate pulse column to determine the effect of frequency on average drop size, back-mixing and coalescence, perhaps with the aid of motion pictures and dyes.

2) Studies be made to determine the effect of pulse height, cam shape and orifice size, shape and spacing on HIU, factors influencing HIU, and flooding.

3) Studies be made to determine the effect of pulsing on spray tower operation and extraction efficiency, with particular emphasis on the mechanism of dispersion and the effect of varied inlet design. 


\section{APPENDIX}

\section{A. Description of Apparatus}

Figure $I$ is a flow diagram of the system and Figures 2-4 are pictures of the experimental equipment. The principal unit, an orifice plate pulse column, was constructed from sections of standard double tough Pyrex pipe, 1-1/2 inches inside diameter. Inlet and outlet connections were made with standard Pyrex pipe tees. The sections were joined with standard metal flanges in the following order: starting from the bottom, 1 tee $\left(7^{n}\right)$, 1 straight (4"), 1 tee $\left(7^{\prime \prime}\right), 18$ straight $\left(72^{\prime \prime}\right), 1$ tee $\left(7^{\prime \prime}\right), 1$ straight $\left(4^{\prime \prime}\right)$, 1 tee ( $\left.7^{\prime \prime}\right)$, and 1 straight (4"). For spray tower operation a single piece of straight 1-1/2 inch inside diameter pipe was substituted for the 18 straight sections.

Teflon gaskets, 1/16" thick, brought the overall height of the column to 114". The internal diameter of the gaskets was 1-1/2" except for the orifice gaskets (19 total, located from the bottom through the top of the $72^{\prime \prime}$ section) which were $3 / 4^{\prime \prime}$. Orifice gaskets, therefore, restricted the area for flow at the joints to $25 \%$ of the column cross-sectional area.

Flanged aluminum plugs tapped internally and externally were used to seal off the tees. No internal fittings were used on the outlet tees, located at the extreme top and bottom of the column. The inlet tees were fitted with $3 / 8^{\prime \prime}$ brass elbows, $90^{\circ}$, to direct flow vertically along the column (see Figure 18). Two brass plates, each containing 25 drilled holes $1 / 16^{\prime \prime}$ in diameter, were soldered to the elbows and acted as distributors.

The pulsing unit (See Figure 3) was driven by a $1 / 6 \mathrm{HP}$ Electro Machine Co. AC motor with a variable speed transmission. The drive frequency range was roughly 0-400 RPM. and was obtained with the aid of suitable pulleys. A $1 / 2^{\prime \prime}$ thick brass circle $2-3 / 4$ " in diameter with a drive shaft displacement of approximately 1/8" served as the cam. These dimensions produced a pulse pattern which was very close to a sine wave (See Figure 19). The cam follower was 


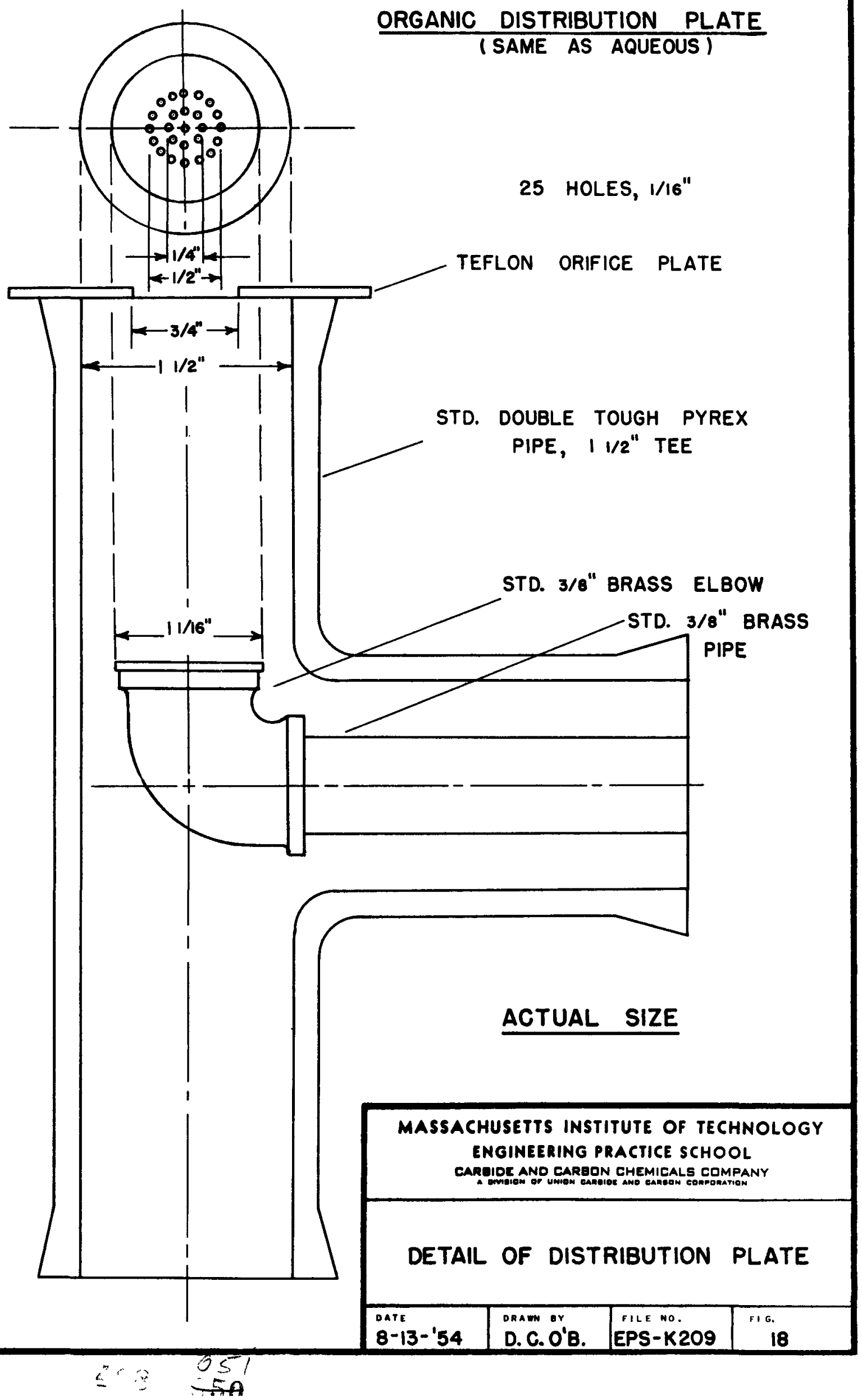




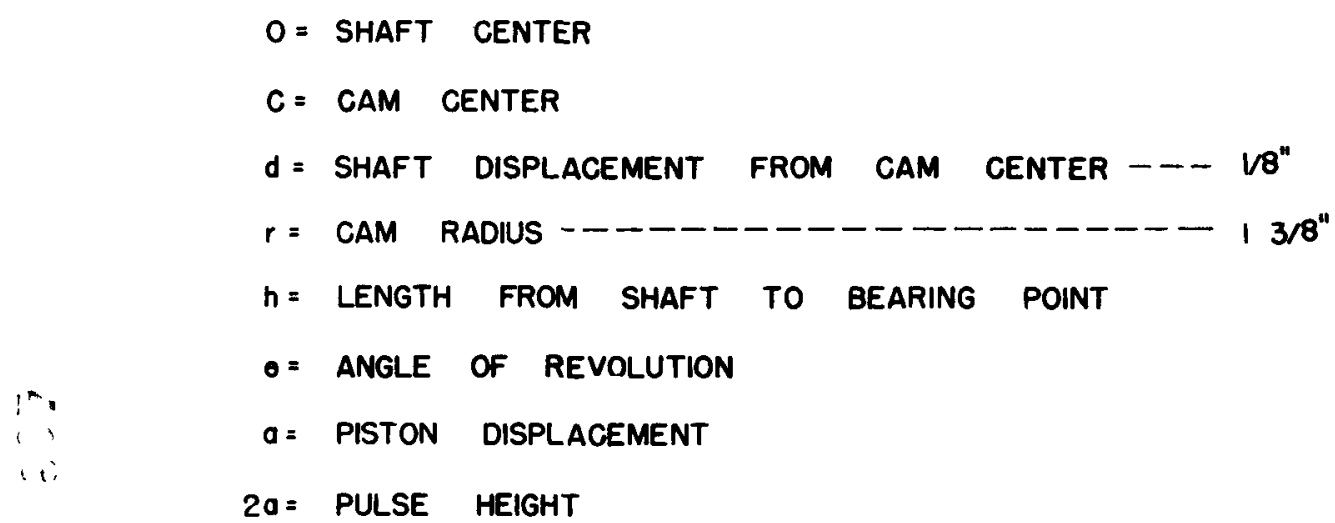

or

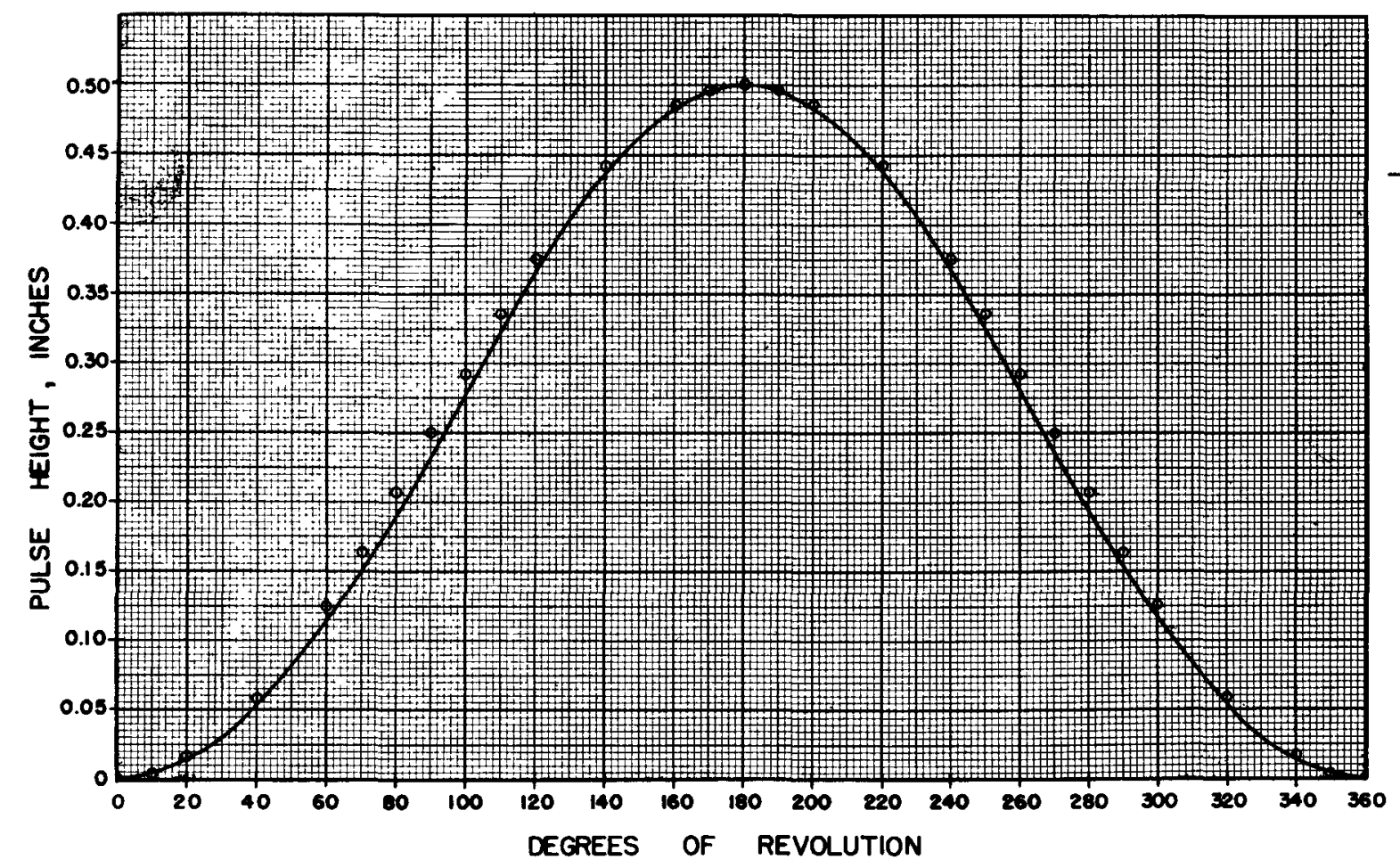

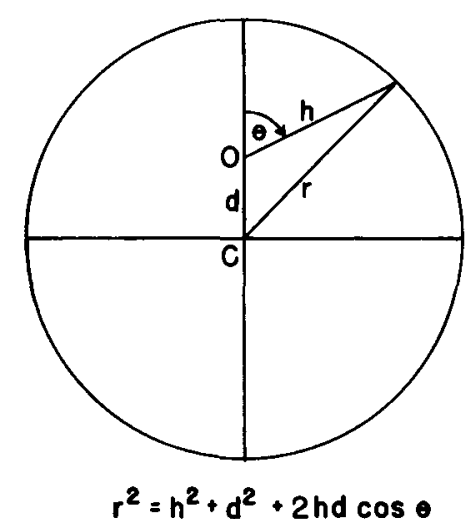

- actual pUlse

- SINE 
a small brass wheel attached to a $1 / 2^{\prime \prime}$ spring-loaded shaft. Brass bellows were fitted to this shaft and were flanged to the bottom of the column. The bellows had the following characteristics: Fulton Sylphon Reference Number 1098, 2-7/16" outside diameter x 1-15/16" inside diameter, 18 active corrugations, one-ply brass, $135 \mathrm{lb} . / \mathrm{sq} .1 \mathrm{n}$. maximum.

Feed and storage tanks were standard bilged 55 gallon single piece stainless steel drums. Three of these drums had 3/8" pipe sections welded through the two top bung caps. One of the sections extended several inches into the drum; the other extended to approximately 2" from the bottom. The shorter pieces of pipe were connected with 1/4" Saran tubing and 1/4" pipe through a reducing valve to a 40 psig. air supply. The longer pieces were fitted to 1/4" Saran tubing and could be interchangeably connected to two rotameters.

The inlet side of each rotameter was constructed with a tee and valve arrangement to allow for feed stream sample removal. Special Fisher-Porter 3A-60-A rotameters were used and were fitted with standard Hoke 1/4" needle valves on the outlet side. The tubing was then connected directly to the respective inlet tees.

The organic outlet was simply an overflow into 1/4" Saran tubing. The aqueous outlet was fitted with a 3/8" Hoke needle valve and consisted of an adjustable, non-syphoning gravity flow liquid leg also made from 1/4" Saran tubing and fittings. Flows were such that both overflows would operate without being completely filled.

\section{B. Chemical System and Analytical Methods}

The chemical system employed was acetic acid, water and hexone (methyl-isobutyl ketone). Factors which made use of this system desirable were: practical regeneration, slight mutual solubility of the two phases, low interfacial tension of hexone, large density difference, and relative ease of analysis. Moreover, use of the system by other investigators allowed comparison of data. Equilibrium data were obtained from Belaga and Bigelow (2) and are shown in Table III.

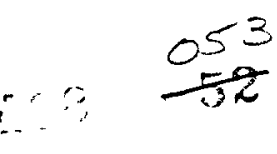


TABLE III

EQUILIBRIUM DATA

(Acetic Acid, Water, Hexone System)

\author{
A (Aqueous Phase) \\ o(Organic Phase)
}

(weight per cent acetic acid)

$\begin{array}{lc}2.95 & 1.79 \\ 6.58 & 4.38 \\ 8.98 & 6.14 \\ 12.11 & 8.77 \\ 1.51 & 0.90 \\ 3.47 & 2.22 \\ 6.61 & 4.47 \\ 9.40 & 6.73 \\ 11.96 & 8.82 \\ 14.55 & 11.09 \\ 16.52 & 12.92 \\ \text { First four points at } 32.5^{\circ} \mathrm{C}, \text { remainder at } 28-29^{\circ} \mathrm{C} .\end{array}$

Source: Belaga, M.W., and Bigelow, J.E., S.M. Thesis, "Effect of Pulse Column Operating Variables on HTU", Report No. KT-133, Oak Ridge, M.I.T. Practice School, Carbide and Carbon Chemicals Co., K-25 (1952).

$$
\begin{array}{r}
054 \\
-\quad: \quad 53
\end{array}
$$


The acid was $99.5 \%$ reagent grade acetic acid produced by J.T. Baker Chemical Co. The water was untreated plant water and the hexone was technical grade methyl-isobutyl ketone purchased from Ansco Solvents and Chemicals Co.

Samples of both phases, ranging in acid concentration from $0.03 \mathrm{~N}$ to $2.3 \mathrm{~N}$, were all titrated with $0.1 \mathrm{~N} \mathrm{NaOH}$ to a phenolphthalein end point. Aliquots were chosen so that roughly $20 \mathrm{ml}$. or more of base were required for neutralization. Generous amounts of water and ethyl alcohol were added to the hexone aliquots to produce a single phase.

\section{Details of Procedure}

The first step in preparing for an extraction run was to make up the aqueous feed. Acid rich water from a previous stripping run was pumped to the feed tank with a small centrifugal pump. In general, it was found that $15 \mathrm{lb}$. of glacial acetic acid would bring the feed up to $2.3 \mathrm{~N}$, the starting concentration. After the contents of the drum was thoroughly stirred, a sample was taken and titrated. Addition and titration was continued until the desired concentration was obtained. Specific gravities were determined with a hydrometer.

A drum of hexone usually lasted for five runs. At the start of a five run series, $200 \mathrm{ml}$. samples were withdrawn from both inlet drums. These samples were assumed to be representative of the entire drum and few checks were taken during a series. The checks always confirmed the assumption of homogeneity.

In beginning an extraction run, the pulsing unit was usually first to be started. Sometimes the pulse height was checked by manually turning the cam to the extreme positions, marking off the change in interface level on a piece of tape and measuring the height with a ruler. Although the cam surface did wear slightly, occasional checks showed that the pulse helght was constant at $1 / 2^{\prime \prime}$. The frequency was then set at the desired level and was determined by counting revolutions with a stopwatch. Checks made during a run rarely showed any change from the original setting. 
After adjusting the air pressure on the displacement pump feed tanks to 8 psig., flow of the continuous phase was started and was adjusted to the predetermined rotameter setting. The interface level was set roughly midway between the continuous phase inlet and the discontinuous phase outlet. Flow of the discontinuous phase was then started and again fixed by the rotameter setting. The interface level was readjusted by raising or lowering the height of the aqueous overflow.

After roughly six or seven changes of the continuous phase, the column was assumed to be at steady-state. Flows of both phases were checked by timing the collection of outlet streams in a $1000 \mathrm{ml}$. graduate with a stopwatch. Hydrometer measurements were made before the two phases were returned to their respective drums. Sometimes part of the material collected for flow rate determination was used as a sample. If not, $200 \mathrm{ml}$. samples were still taken at the same time, but directly from the outlet lines. The same procedure was repeated 30 minutes later as a check on steady-state.

The procedure for ending a run varied depending upon which phase was discontinuous. If the aqueous was discontinuous, the aqueous outlet and inlet valves were shut as quickly as possible. The organic flow was allowed to continue but the pulser was stopped. After the phases were separated, the aqueous outlet line was opened and the interface level was allowed to drop to the operating level. The aqueous overflow was collected and was taken as the holdup.

If the organic phase was discontinuous, the interface level was dropped and both inlet flows were shut off as quickly as possible. This operation stopped the organic outflow. The aqueous outlet line was then shut. After stopping the pulser and allowing the phases to separate, aqueous phase was fed to the column until the interface had climbed to the normal operating level. The organic overflow was collected and was taken as the holdup.

At the end of an extraction run series the aqueous raffinate was discarded. The acid-rich organic tank was connected to the air

$$
\begin{array}{r}
056 \\
75 \\
\hline 50
\end{array}
$$


line and the organic rotameter. The aqueous rotameter was connected directly to the plant water line, and the column was then ready to strip. Operating procedure was the same for extraction except that normally only one run was taken per drum.

As noted before, rotameters were used primarily for setting flow rates but not for measuring them. For flooding studies, however, rotameters were used for flow rate measurement due to the transient nature of the total discontinuous phase holdup. They were calibrated by using flow data from the most recent runs. The plots of rotameter setting versus solute-free flow rate based on outlet flow rate proved to be fairly good straight lines. Rotameter settings for flooding studies were chosen at intervals of approximately $40 \mathrm{gm}$. $/ \mathrm{min}$. solutefree aqueous flow at a $Q_{0} / Q_{a}$ of 1.33 .

In flooding studies, the operating procedure was much the same as in other runs. At a given frequency, rotameter settings were changed from low to high values. The lowest setting at which flooding occurred was called the flooding point.

Aqueous discontinuous flooding was defined as the throughput at which not all of the aqueous phase could pass through the first orifice, but was partially forced out the organic outlet. Evidence of this type of flooding was seen as a secondary interface which formed at the aqueous inlet and tended to rise to the top of the column. The heavier phase actually consisted of an emulsified mixture of hexone and water. Such a formation frequently did not take place immediately, and consequently 15 minutes was allowed at each setting.

Organic discontinuous flooding was more arbitrary in definition. It was defined as the throughput at which the organic coalesced to form large drops to such an extent that it nearly filled a cell and became the continuous phase in that cell. 


\section{Sample Calculations}

Run No. 65 was chosen as a basis for the sample calculation.

1. Column Characteristics:

Effective height - 85"

Plate spacing - 4"

Orifice diameter - 3/4"

2. Operating Conditions:

Discontinuous phase

organic

Transfer

aqueous to organic

Volume in effective extraction

height

Holdup

Room Temperature
$2350 \mathrm{ml}$. $690 \mathrm{ml}$. $81^{\circ} \mathrm{F}$.

3. Flow Rates:

Aqueous

Sample 1

Sample 2

Organic

Sample 1

Sample 2

4.

Inlet Aqueous

Inlet Organic

Outlet Aqueous: Sample 1

Sample 2

Outlet Organic: Sample 1

Sample 2 ml. collected

992

990

1002

996

Specific Gravity*
Time (sec.)

189.6

189.0

112.6

111.0

Normality

1.011

2.307

0.799

0.0364

0.998

0.569

0.998

0.566

0.826

1.272

0.826

* No temperature correction was made for the hydrometer.

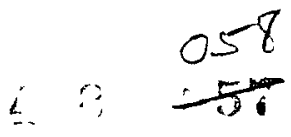


5. Concentrations of the four streams were calculated using averaged normalities as follows:

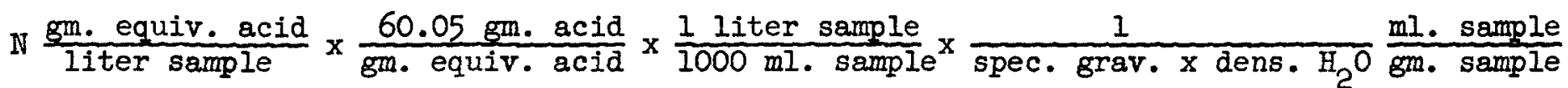

$$
=\frac{\text { gm. acid }}{\text { gm. sample }}
$$

Inlet Aqueous: $A_{1}=\frac{(2.307)(60.05)}{(1000)(1.011)}=0.1370 \frac{\mathrm{gm} \cdot \text { acid }}{\mathrm{gm} \cdot \text { sample }}$

Inlet Organic: $o_{2}=\frac{(0.0364)(60.05)}{(1000)(0.799)}=0.0027 \frac{\text { gm. acid }}{\text { gm. sample }}$

Outlet Aqueous: $A_{2}=\frac{\left(\frac{0.569+0.566}{2}\right)(60.05)}{(1000)(0.998)}=0.0342 \frac{\text { gm. acid }}{\text { gm. sample }}$

Outlet Organic: $o_{1}=\frac{\left(\frac{1.272+1.247}{2}\right)(60.05)}{(1000)(0.826)}=0.0916 \frac{\mathrm{gm} \cdot \text { acid }}{\mathrm{gm} \cdot \mathrm{smple}}$

6. These concentrations were converted to gm. acid/gm. acid-free solvent, and the corresponding equilibrium values were obtained from a plot of $X^{*}$ vs. $Y^{*}$ for this system (See Figure 20):

Inlet Aqueous $X_{1}=\frac{A_{1}}{I-A_{1}}=\frac{0.1370}{0.8630}=0.1587 \quad \frac{\text { Values }}{Y_{1}^{*}=0.1130}$

Inlet Organic $Y_{2}=\frac{O_{2}}{1-O_{2}}=\frac{0.0027}{0.9973}=0.0028 \quad X_{2}^{*}=0.0042$

Outlet Aqueous $X_{2}=\frac{A_{2}}{I-A_{2}}=\frac{0.0342}{0.9658}=0.0354 \quad Y_{2}^{*}=0.0229$

Outlet Organic $Y_{1}=\frac{O_{1}}{I-O_{1}}=\frac{0.0916}{0.9084}=0.1008 \quad X_{1}^{*}=0.1438$ 


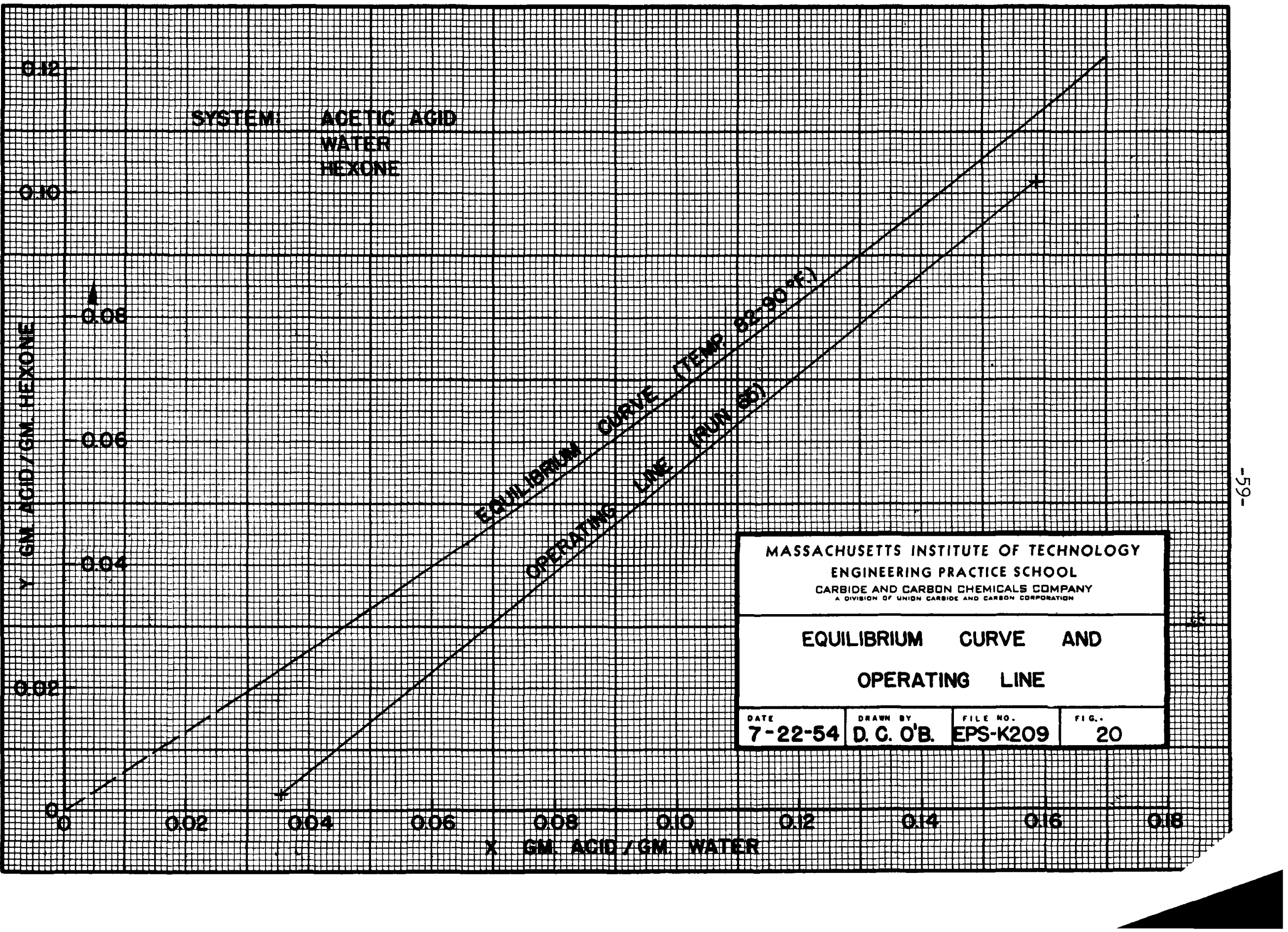


7. Solute-free flow rates vere calculated as follows;

$Q=\frac{\mathrm{ml} \cdot \text { sample collected }}{\text { sec. }} \times \frac{60 \mathrm{sec}}{\mathrm{min} \cdot} \times\left(\mathrm{sp} \cdot \mathrm{g} \cdot \mathrm{x}\right.$ dens. $\left.\mathrm{H}_{2} \mathrm{O}\right) \frac{\mathrm{gm} \cdot \mathrm{sample}}{\mathrm{ml} \cdot \mathrm{sample}} \times \frac{\mathrm{gm} \cdot \text { solute-free solvent }}{\mathrm{gm} \cdot \mathrm{sample}}$

$=\frac{\text { gm. solute-free solvent }}{\min .}$

Outlet Aqueous:

Sample 1: $Q_{a}=\frac{992}{189.6} \times 60 \times 0.998 \times 0.966=303 \frac{\text { gm. solute-free solvent }}{\text { min. }}$

Sample 2: $Q_{a}=\frac{990}{189} \times 60 \times 0.998 \times 0.966=303 \frac{\text { gm. solute-free solvent }}{\text { min. }}$ Average $Q_{a} \quad=\quad 303 \frac{\mathrm{gm} \text {. solute-free solvent }}{\min .}$

Outlet Organic:

Sample 1: $Q_{0}=\frac{1002}{112.6} \times 60 \times 0.826 \times 0.908=400 \frac{\mathrm{gm} \text {. solute-free solvent }}{\mathrm{min} .}$ Sample $2: Q_{0}=\frac{996}{111.0} \times 60 \times 0.826 \times 0.908=404 \frac{\mathrm{gm} . \text { solute-free solvent }}{\min .}$ Average $Q_{0}=402 \frac{\text { gm. solute-free solvent }}{\text { min. }}$

8. Ratio of solute-free organic flow to solute free aqueous flow:

$$
Q_{0} / Q_{a}=\frac{402}{303}=1.33
$$

9. Material balance on acid:

Acid in: Inlet Aqueous: $\left(Q_{a} \times x_{1}\right)=303 \times 0.1587=48.1 \frac{\mathrm{gm} .}{\mathrm{min}}$. Inlet Organic: $\left(Q_{0} \times Y_{2}\right)=402 \times 0.0028=1.1 "$ Total acid in $49.2 \frac{\mathrm{gm}}{\mathrm{min}}$.

Acid out: Outlet Aqueous: $\left(\mathrm{Q}_{\mathrm{a}} \times \mathrm{x}_{2}\right)=303 \times 0.0354=10.7 \frac{\mathrm{gm} .}{\mathrm{min}}$. Outlet Organic: $\left(Q_{0} \times Y_{1}\right)=402 \times 0.1008=40.5 "$ Total acid out $\overline{51.2} \frac{\mathrm{gm} .}{\mathrm{min}}$.

Per cent deviation based on acid in:

$$
\left(\frac{51.2-49.2}{49.2}\right) 100=+4.1 \%
$$


1.0. Evaluation of $\mathrm{HTU}_{\mathrm{OO}}$ and $\mathrm{HTU}_{\mathrm{Oa}}$

The following assumptions were made:

$$
\begin{aligned}
& \mathrm{NTU}_{00}=\int_{Y_{1}}^{Y_{2}} \frac{d Y}{Y^{*}-Y} \cong \frac{Y_{2}-Y_{I}}{\left(Y^{*}-\bar{Y}\right)_{L \text { Iog mean }}} \\
& \mathrm{NIU}_{\text {oa }}=\int_{\mathrm{X}_{1}}^{\mathrm{x}_{2}} \frac{\mathrm{dx}}{\bar{x}-\mathrm{X}^{*}} \cong \frac{\mathrm{x}_{1}-\mathrm{x}_{2}}{\left(\bar{x}-\mathrm{X}^{*}\right)_{\text {log mean }}}
\end{aligned}
$$

Calculations were made as follows:

NTU

NT⿱卄一由八

$$
\begin{aligned}
& Y_{2}-Y_{1}=0.1008-0.0028=0.0980 \\
& \left(Y^{*}-Y_{\log \text { mean }}=\frac{\left(Y^{*}-Y_{2}-\left(Y^{*}-Y\right)_{1}\right.}{\ln \frac{\left(Y^{*}-Y\right)_{2}}{\left(Y^{*}-Y\right)_{1}}}\right. \\
& =\frac{(0.1130-0.1008)-(0.0229-0.0028)}{\ln \frac{(0.1130-0.1008)}{(0.0229-0.0028)}} \\
& =0.0159 \\
& \mathrm{NWU}_{00}=\frac{0.0980}{0.0159}=6.16 \\
& \mathrm{HTU}_{\mathrm{OO}}=\frac{\text { Effective column height }}{\text { NTU }}=\frac{85^{\prime \prime}}{6.16}=13.8^{\prime \prime}
\end{aligned}
$$

NTU

oa

$$
\begin{aligned}
\mathrm{X}_{1}-\mathrm{X}_{2}=0.1587 & -0.0354=0.1233 \\
\left(\mathrm{X}-\mathrm{X}^{*}\right)_{\log \text { mean }} & =\frac{\left(\mathrm{X}-\mathrm{X}^{*}\right)_{1}-\left(\mathrm{X}-\mathrm{X}^{*}\right)_{2}}{\left(\mathrm{X}-\mathrm{X}^{*}\right)_{1}} \\
\ln \frac{\left(\mathrm{X}-\mathrm{X}^{*}\right)_{2}}{(0.1587-0.1438)-(0.0354-0.0042)} & \frac{\ln \frac{(0.1587-0.1438)}{(0.0354-0.0042)}}{} \\
& =0.0220
\end{aligned}
$$




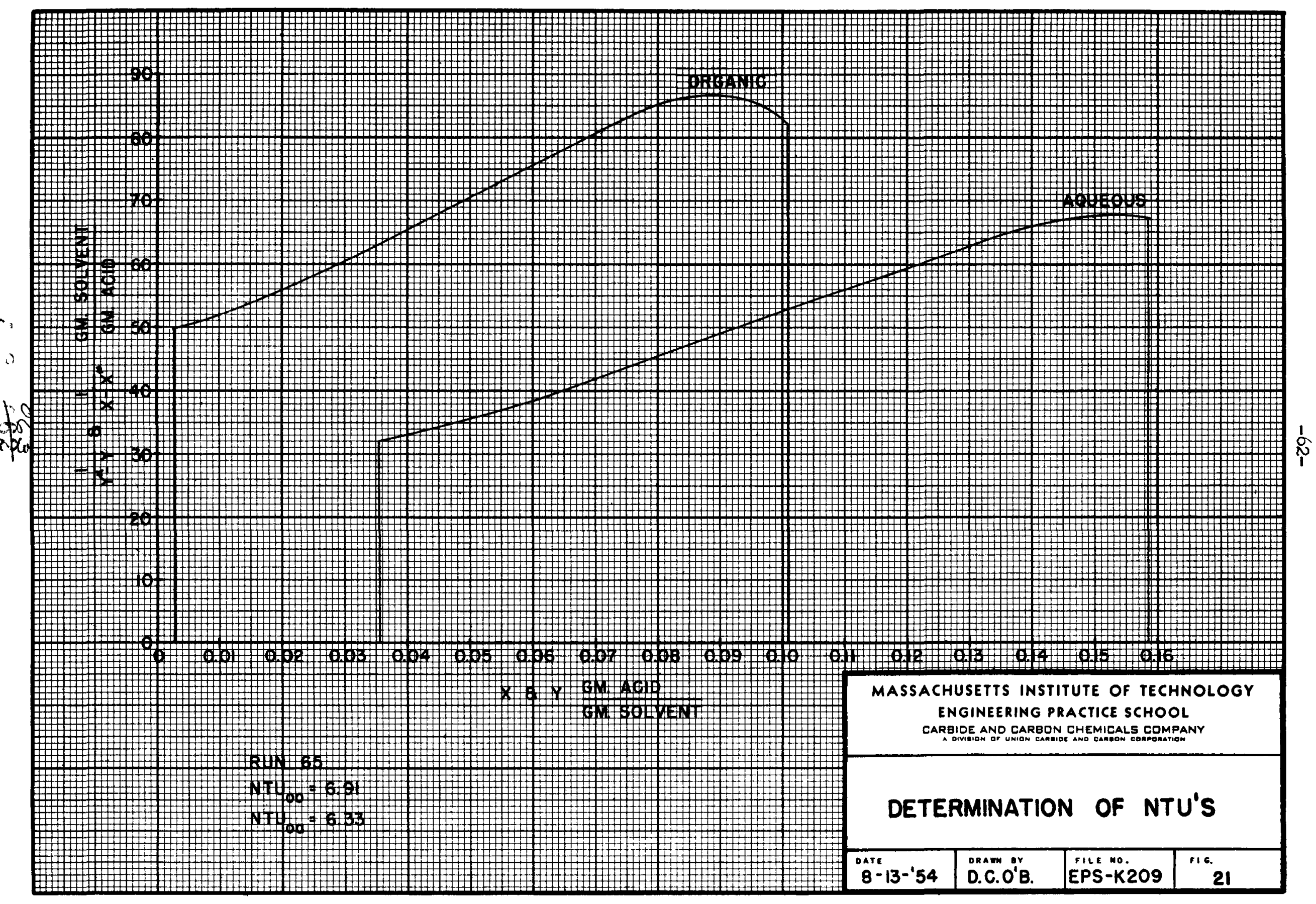




$$
\begin{aligned}
& \mathrm{NIU}_{\mathrm{OQ}}=\frac{0.1233}{0.0220}=5.60 \\
& { }_{\text {HTU }}=\frac{\text { Effective column height }}{\text { NIU }}=\frac{85^{\prime \prime}}{5.60}=15.2^{\prime \prime}
\end{aligned}
$$

11. More accurate determinations of $\mathrm{NIU}_{\mathrm{OO}}$ and $\mathrm{NIU} \mathrm{Oa}_{\text {were made by }}$ graphical integrations of plots of $Y$ vs. $I\left(Y^{*}-Y\right)$ and $X$ vs. $I\left(X-X^{*}\right)$ (See Figure 21).

The following values were obtained:

$$
\begin{aligned}
& \mathrm{NIU}_{\mathrm{OO}}=6.91 \quad \text { HTU }_{O O}=\frac{85^{\prime \prime}}{6.91}=12.3^{\prime \prime} \\
& \mathrm{NTU}_{\mathrm{OQ}}=6.33 \quad \mathrm{HTU}_{\mathrm{OQ}}=\frac{85^{\prime \prime}}{6.33}=13.4^{\mathrm{n}}
\end{aligned}
$$

12. The errors introduced by the assumption of log-mean driving force were:

$$
\begin{aligned}
& \text { NIU } 00 \\
& \text { : }\left(\frac{6.91-6.16}{6.91}\right) \times 100=10.8 \% \\
& \text { NIU }_{O Q}:\left(\frac{6.33-5.60}{6.33}\right) \times 100=11.5 \%
\end{aligned}
$$

13. Similar calculations for run No. 41 showed:

$$
\begin{aligned}
& \mathrm{NIU}_{\mathrm{OO}}(\text { log mean })=3.56 \quad \mathrm{HTU}_{\mathrm{OO}}=23.9^{\prime \prime} \\
& \mathrm{NTU}_{\mathrm{OQ}}(\log \text { mean })=3.18 \quad \text { HTU }_{\mathrm{Oa}}=26.7^{\prime \prime} \\
& \text { NTU }_{\text {Oo }} \text { (graphical integration) }=3.79 \\
& \mathrm{NIU}_{\text {oa }} \text { (graphical integration) }=3.38
\end{aligned}
$$

The errors introduced by the assumption of log-mean driving force were:

$$
\begin{aligned}
& \mathrm{NTU}_{00}:\left(\frac{3.79-3.56}{3.79}\right) \times 100=6.1 \% \\
& \mathrm{NTU}_{\mathrm{OQ}}:\left(\frac{3.38-3.18}{3.38}\right) \times 100=5.9 \%
\end{aligned}
$$

It is seen from these two runs that the error tends to decrease with increasing HIU.

$$
\therefore=\frac{064}{-33}
$$


14. Discontinuous organic holdup in terms of volume per cent was (for run No. 65):

$$
\left(\frac{690}{2350}\right) \times 100=29.4 \% \text { holdup }
$$

E. Summary of Data and Calculated Values

Table IV is a summary of data and calculated values.

$$
\therefore=065
$$


Table IV

SUMMARY OF DATA AND CALCULATED VALUES

OFIFICE PLATS COLUMN

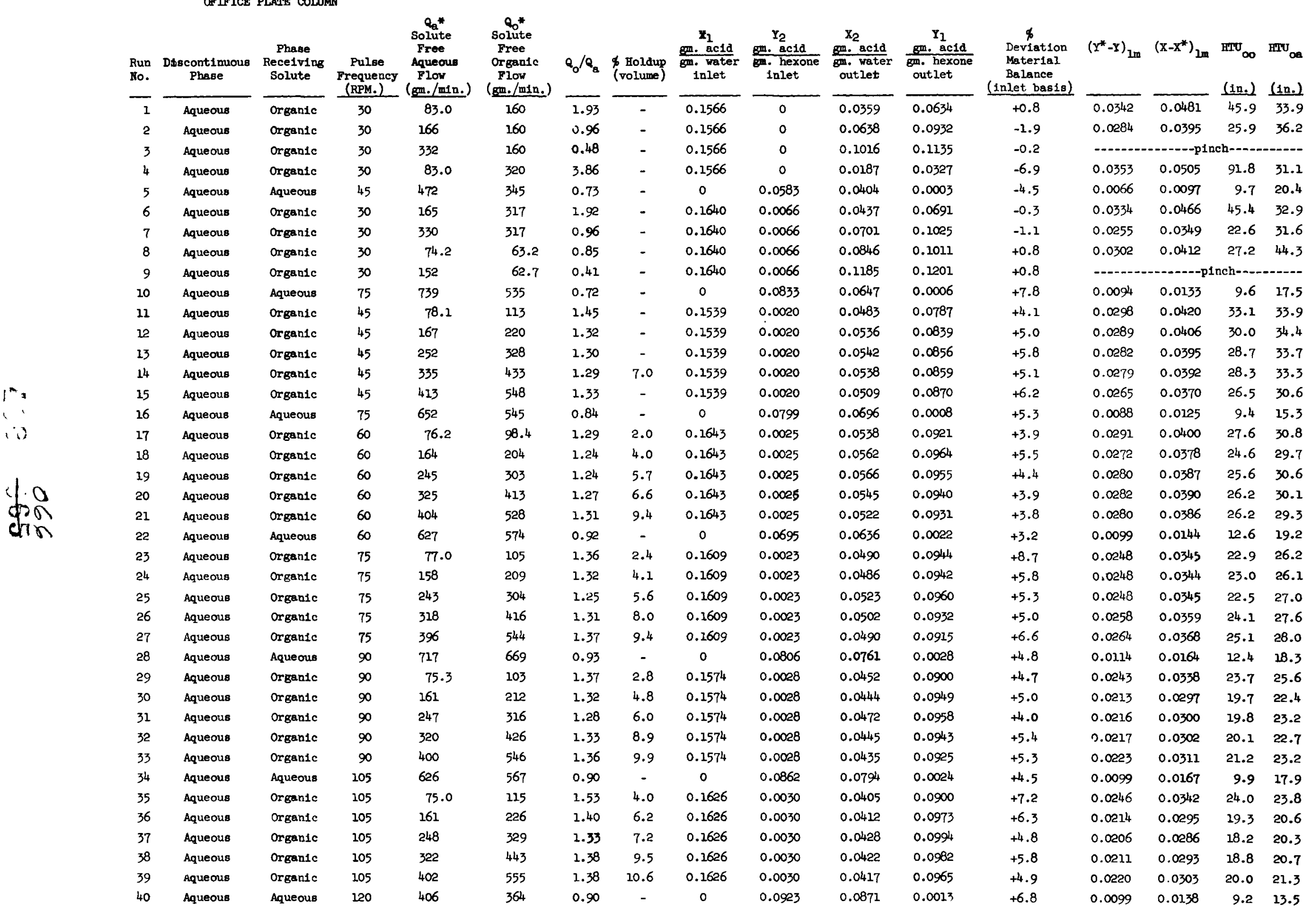

* Flows for Runs 1-7 based on rotameter calibrations. 
Table IV (Cont'd.)

SUMMARY OF DATA AND CALCULATED VALUES

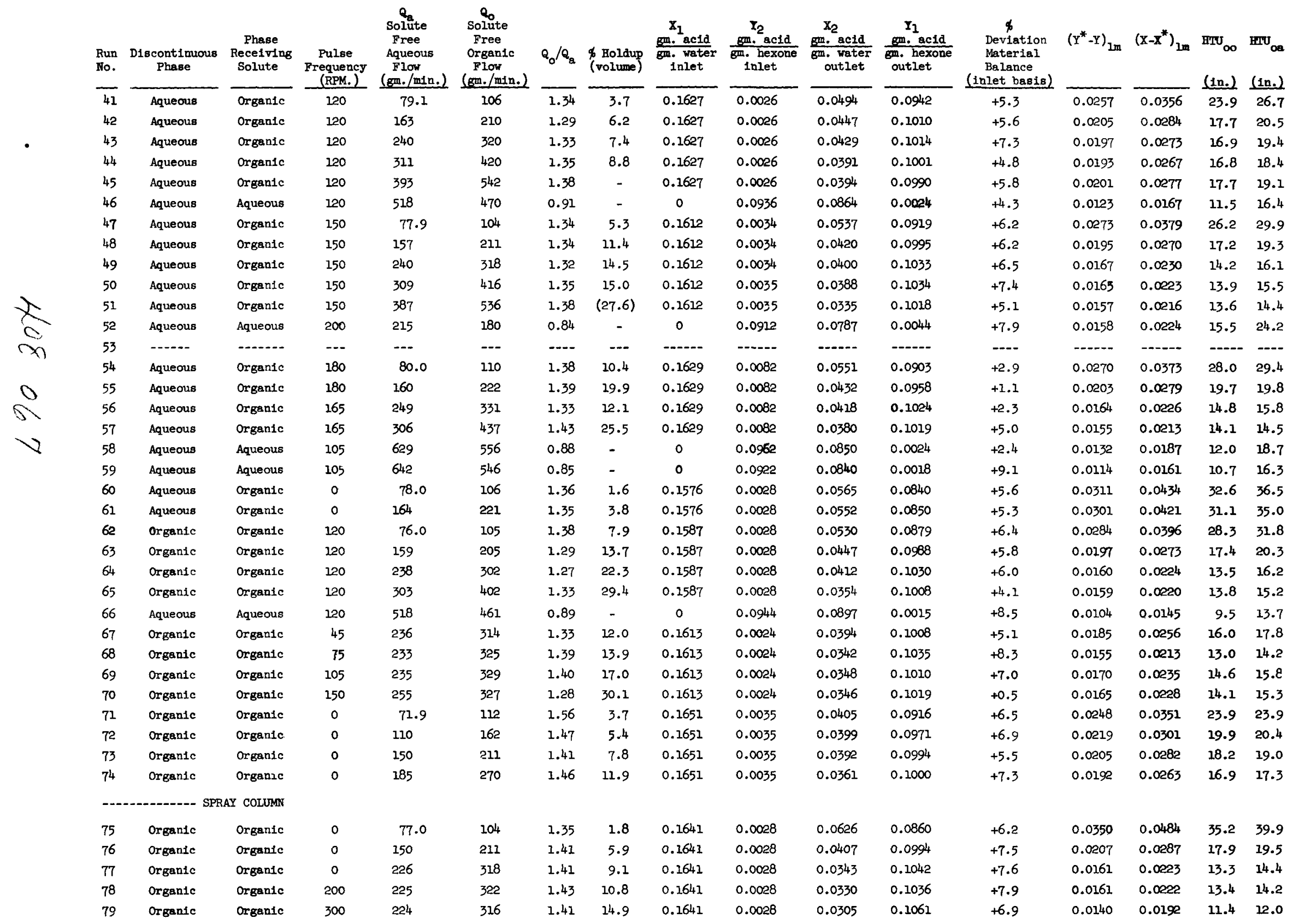


F. Table of Nomenclature

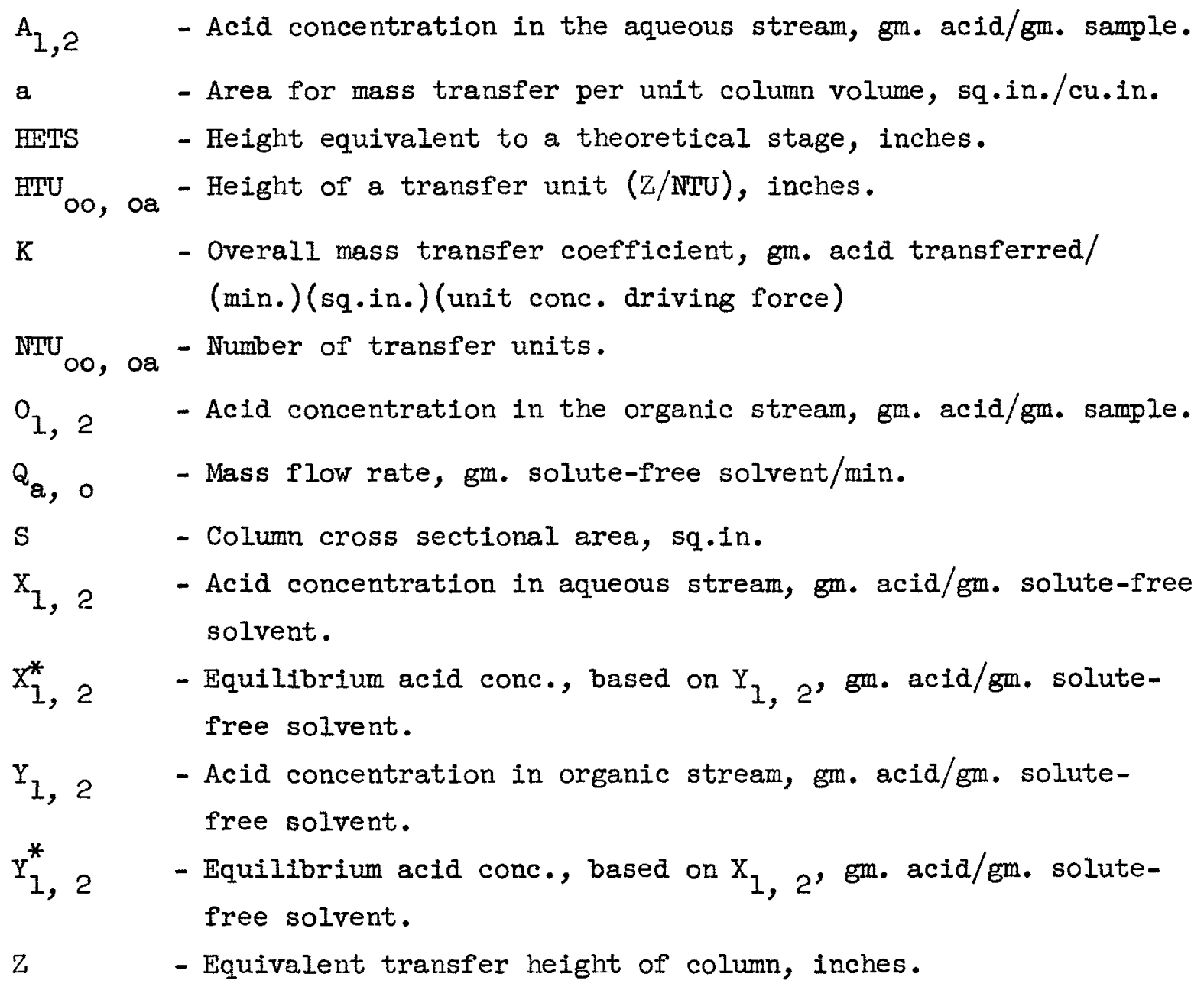

Subscripts :

1 refers to top of column, aqueous inlet and organic outlet.

2 refers to bottom of column, aqueous outlet and organic inlet.

a refers to aqueous phase

o refers to organic phase

oa refers to overall based on aqueous phase

oo refers to overall based on organic phase

G. Location of Original Data and Calculations

The original data are found in part on pages $61-96$, Data Book No. 2487 . The data book, the remainder of the data and the calculations may be found at the M.I.T. Engineering Practice School, Oak Ridge, Tennessee. 
H. Literature Citations

(1) Baker, M.L., and Lang, P.M., "Effects of Flow Rates on Pulse Column Operation", Report No. EPS-K-162, Oak Ridge, M.I.T. Practice School, Carbide and Carbon Chemicals Co., K-25 (1951).

(2) Belaga, M.W., and Bigelow, J.E., S.M. Thesis, "Effect of Pulse Column Operating Variables on HTU", Report No. Kr-133, Oak Ridge, M.I.T. Practice School, Carbide and Carbon Chemicals Co., K-25 (1952).

(3) Buchanan, J.R., Lueders, R.E., Reynolds, A.B., and Stewart, W.A., "Uranium Recovery Using Sieve Plate and Orifice Plate Pulse Columns", Report No. KT-171, Oak Ridge, M.I.T. Practice School, Carbide and Carbon Chemicals Co., K-25 (1954).

(4) Coplan, B.V., and Davidson, J.K., "Solvent-Extraction Process Contacting Equipment", Reactor Science and Technology, 3, 1, USAEC, Mar., 1953.

(5) Fleming, J.F., and Johnson, H.F., "Liquid-Liquid Extraction at High Flow Rates in a Spray Tower", Chemical Engineering Progress, 49, 9, 497-509, Sept., 1953.

(6) Gavin, J.V., and Thies, C.E., "Effect of Plate Spacing in Pulse Columns", Report No. KT-152, Oak Ridge, M.I.T. Practice School, Carbide and Carbon Chemicals Co., K-25 (1952).

(7) Lurie, R.M., and Shaver, R.G., "The Effect of Vibration on LiquidLiquid Extraction", Report No. KT-149, Oak Ridge, M.I.T. Practice School, Carbide and Carbon Chemicals Co., K-25 (1952).

(8) McCorkle, K.M., Erickson, A.J., and Wasserman, A.A., "Investigation of Holdup in a Pulse Column", Report No. EPS-K-161, Oak Ridge, M.I.T. Practice School, Carbide and Carbon Chemicals Co., K-25 (1951).

(9) Myers, J.H., O'Brien, D.C., Hutchins, A.G., and Skinner, F., "Uranium Extraction From Slurries", Report No. EPS-Y-196, Oak Ridge, M.I.T. Practice School, Carbide and Carbon Chemicals Co., K-25 (1953). 
(10) Perry, J.H., editor, Chemical Engineers' Handbook, 3rd Ed., 748-753, 1019, McGraw-Hill, New York, 1950.

(11) Reuther, R.H., Barlow, J.W., and Morgenthaler, J.H., "Investigation of Pulse Column Variables", Report No. EPS-K-153, Oak Ridge, Carbide and Carbon Chemicals Co., K-25 (1951).

(12) Weigandt, H.F., and VonBerg, J.I., "Key to Better Extraction", Chemical Engineering, 61, 7, 183-188, July, 1954. 